OUTP 0611P

\title{
Probing neutrino oscillations from supernovae shock waves via the IceCube detector
}

\author{
Sandhya Choubey ${ }^{1,2 *}$, N. P. Harries ${ }^{1 \dagger}$, G.G. Ross ${ }^{1 \ddagger}$ \\ ${ }^{1}$ The Rudolf Peierls Centre for Theoretical Physics, \\ University of Oxford, 1 Keble Road, Oxford, OX1 3NP, UK \\ ${ }^{2}$ Harish-Chandra Research Institute, \\ Chhatnag Road, Jhunsi, Allahabad 211 019, INDIA
}

\begin{abstract}
The time dependent neutrino oscillation signals due to the passage of a shock wave through the supernovae are analyzed for the case of three active neutrinos and also for the case that there are two additional sterile neutrinos. It is shown that, even without flavour identification and energy measurement, detailed information about the masses and mixing angles of the neutrinos may be obtained with a detector with excellent time resolution such as IceCube. Such a signal would also give important information about the nature of the shock wave within the supernovae.
\end{abstract}

\section{Introduction}

The possibility that, in addition to the three flavours of light neutrinos required by the Standard Model, there are light sterile neutrinos has been severely constrained by neutrino oscillation experiments. However there is still the outstanding problem of explaining the data from the Liquid Scintillator Neutrino Detector (LSND) [1] which, if confirmed, might require the existence of at least one extra light neutrino. The precision measurements at LEP have shown that only three neutrinos with standard weak interactions exist with mass less than $M_{Z} / 2$ and therefore additional neutrinos must be sterile [2]. However the addition of a single sterile neutrino to

*email: sandhya@mri.ernet.in

$\dagger$ email: n.harries1@physics.ox.ac.uk

$\ddagger$ email: g.ross1@physics.ox.ac.uk 
explain the LSND anomaly is not able to give a good fit to solar and KamLAND [3, 4] and atmospheric [5, 6] data together with the data from the short-baseline experiments CHOOZ 7] Bugey, CCFR84, CDHS, KARMEN and NOMAD [8]. While a $3+1$ hierarchy in the four neutrino case is preferred over a $2+2$ hierarchy, neither is acceptable 9] and one is driven to consider the case of two additional sterile neutrinos [10. In this case a $3+2$ mass hierarchy gives an acceptable fit, significantly better than that found for the $3+1$ case.

Pinning down the properties of additional sterile neutrinos is a daunting task. Even if the LSND result is not confirmed there will still be room for light sterile neutrinos mixing with the three active states that terrestrial experiments will not be able to exclude. Here we investigate the possibility of gaining significant new information on this sector from the neutrinos coming from supernovae. The reason this can give a significant signal is due to the possibility of resonant conversion of the various neutrino species (the Mikheyev-Smirnov-Wolfenstein (MSW) effect [11]) within the supernova. The MSW effect, being a resonant process, is much more sensitive to small mixing angles than non resonant oscillation phenomena. Feasibility studies of using neutrino signal from a galactic supernova to determine the neutrino mass hierarchy and constrain the mixing angle $\theta_{13}$ have been done before in [12 for the standard case of three active neutrinos and in [13] for three active and one sterile neutrino.

A further significant advantage of the supernovae signals for neutrino oscillation has been appreciated in recent years [14. This follows from the fact that in the supernova explosion a shock wave propagates through the supernova. From the theoretical simulations of supernovae it is believed that when the core of the collapsing star reaches nuclear density, the collapse rebounds forming a strong outward shock. This is stalled and then regenerated by a neutrino driven wind. During this process it is believed that as well as a forward shock a reverse shock could also form [15. At the shock front the density changes very rapidly so that the resonant transition may become non-adiabatic as the shock wave passes [14, 15 $]^{1}$. As a result one may have rapid changes in the active neutrino luminosity produced by the supernova, very characteristic signals which do not require knowledge of the overall luminosity. These signals are observable by detectors such as IceCube ${ }^{2}$ capable of collecting high statistics with excellent time resolution. IceCube will provide a $\mathrm{km}^{3}$ neutrino detector with a time resolution of 10ns [17]. Although designed to observe high energy neutrinos from astrophysical sources, it has been realized that the lower energy neutrinos from supernovae can be probed in IceCube through the detection of additional photons in the background halo, caused by supernovae neutrinos interacting with the ice [21]. IceCube cannot measure the energy spectra of the supernova neutrinos, but because of its excellent time resolution and high statistics, we find, for the reasons given above, that it can be an excellent probe of a sterile neutrino component to neutrino mass eigenstates. Moreover observation of such signals in effect provide information on supernovae seismology and can yield important information on the nature of the shock wave within the supernovae.

For the case of oscillation between active (anti)neutrinos only, detection of such effects requires a difference in the initial properties of the neutrinos produced at the neutrinosphere. However there is considerable uncertainty in this. Early studies [22] suggested that, while the luminosities of the three antineutrino species should be quite close, the average energy of the $\bar{\nu}_{e}$

\footnotetext{
${ }^{1}$ While completing this paper we received a paper [16] studying the effects of turbulence on the shock wave. These effects have not be included here but may be expected to broaden the structures presented below.

${ }^{2}$ For detailed discussion of the effect of the shock wave on the signal in water Cerenkov detectors, we refer the readers to [14, 18, 19] for effects of the forward shock only and [15, 20] for both forward and reverse shocks.
} 
should be about half that of $\bar{\nu}_{\mu}$ and $\bar{\nu}_{\tau}$. More recently studies 23] including additional scattering processes within the neutrinosphere have suggested that the difference in the average energies of the $\bar{\nu}_{e}$ and $\bar{\nu}_{\mu} / \bar{\nu}_{\tau}$ is quite small but that there is a significant difference in their luminosities. We will investigate the time dependent signals for active neutrino oscillation for both these cases. For the case of oscillation to sterile neutrinos the time dependent oscillation signals are not so sensitive to uncertainties in the initial antineutrino processes because sterile neutrinos produced by resonant oscillation will not be visible in detectors and so oscillation to a sterile neutrino is observable even with a detector having no capability to distinguish the flavour or energy of active neutrinos.

In this paper we study the signals to be expected by IceCube both for the case of just three active neutrinos and for the case that there are two additional sterile neutrinos. To make the discussion tractable we concentrate on the range of mass and mixing angle parameters that provide an explanation of the LSND events while being consistent together with all other neutrino oscillation data. However our results indicate that such signals will also be significant in constraining the sterile neutrino component even if the LSND result is not confirmed.

In Section 2 we briefly review resonant conversion within the supernova, the various possibilities for the neutrino spectra and list the parameters used in the subsequent analysis. In Section 3 we discuss the properties of the neutrinos at the neutrinosphere and the nature of the shockwaves propagating through the supernova. Section 4 contains the results of our analysis. We first review the characteristics of the IceCube detector and then present its detection rates for a "standard" supernova for the case of three active neutrinos only and for the case of three active neutrinos plus two sterile neutrinos for the various possible neutrino mass hierarchy possibilities. Finally in Section 5 we present our conclusions.

\section{Resonant Conversion Within the Supernova}

\subsection{Three Active Neutrinos}

We start with the case of three active (anti)neutrino species. In the basis of flavor eigenstates the evolution of (anti)neutrinos is described by the effective Hamiltonian

$$
\mathcal{H}=\frac{1}{2 E}\left(U \mathcal{M}^{2} U^{\dagger}+\mathcal{A}\right)
$$

where $U$ is a unitary matrix and is defined by $\left|\nu_{i}\right\rangle=\sum_{\alpha} U_{i \alpha}\left|\nu_{\alpha}\right\rangle$. The vacuum mass eigenstates are $\nu_{i}$ and the weak interaction eigenstates are $\nu_{\alpha} . \mathcal{M}^{2}$ is the matrix of mass squares of the mass eigenstates in vacuo, and $\mathcal{A}$ is the matter induced mass matrix. For mixing between three active neutrinos

$$
\begin{gathered}
\mathcal{M}^{2}=\operatorname{Diag}\left(m_{1}^{2}, m_{2}^{2}, m_{3}^{2}\right), \\
\mathcal{A}=\operatorname{Diag}\left(A_{1}, 0,0\right), \\
A_{1}= \pm \sqrt{2} G_{F} \rho N_{A} Y_{e} \times 2 E,
\end{gathered}
$$



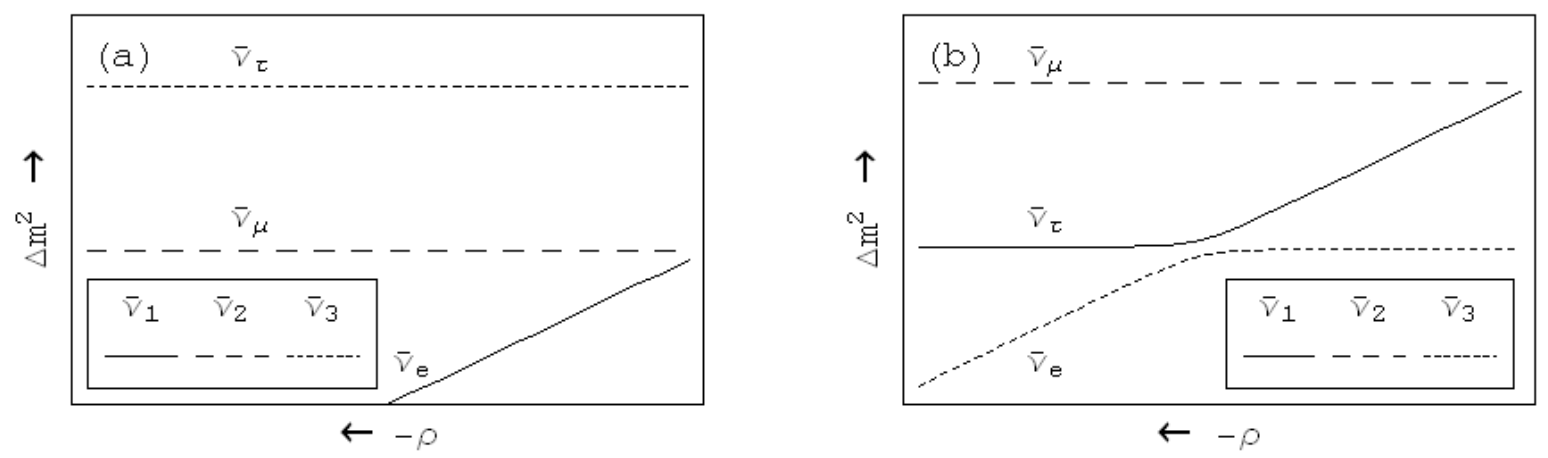

Figure 1: The evolution with density of the mass eigenvalues (squared) for the case of three active antineutrinos (a) for the normal hierarchy $\left(\Delta m_{31}^{2}>0\right)$ and (b) for the inverse hierarchy $\left(\Delta m_{31}^{2}<0\right)$.

where $i=1-3$ and $\alpha=e, \mu$ or $\tau, m_{i}^{2}$ is the mass squared of the $i^{\text {th }}$ vacuum mass eigenstate, $G_{F}$ is the Fermi coupling constant, $\rho$ is the density of ambient matter, $N_{A}$ is the Avogadro's number, $Y_{e}$ is the electron fraction and $E$ is the energy of the neutrino. $A_{1}$ is the matter potential induced by charged current interactions of $\nu_{e}\left(\bar{\nu}_{e}\right)$ with electrons and the "+" ("-") sign in Eq. (41) corresponds to neutrinos (antineutrinos). There is also an effective mass squared induced by neutral current interactions of the (anti)neutrinos with matter. However, this does not have a physical effect because the neutral current interaction is universal for all (anti)neutrino flavours, while oscillations depend on the difference of mass squared and not on the absolute value. The mixing matrix $U$ is given in terms of mixing angles and CP-violating phases. If $\mathrm{CP}$ conservation is assumed the mixing matrix takes the form

$$
U=\prod_{0<B<A} \prod_{A=2}^{3} R^{B A}\left(\theta_{A B}\right)
$$

where $R^{A B}\left(\theta_{A B}\right)$ are rotation matrices, representing a rotation of $\left(\theta_{A B}\right)$ in the AB plane. The mixing matrix for three flavours is therefore given as

$$
U=\left(\begin{array}{ccc}
c_{12} c_{13} & s_{12} c_{13} & s_{13} \\
-s_{12} c_{23}-c_{12} s_{23} s_{13} & c_{12} c_{23}-s_{12} s_{23} s_{13} & s_{23} c_{13} \\
s_{12} s_{23}-c_{12} c_{23} s_{13} & -c_{12} s_{23}-s_{12} c_{23} s_{13} & c_{23} c_{13}
\end{array}\right)
$$

where $c_{i j} \equiv \cos \theta_{i j}$ and $s_{i j} \equiv \sin \theta_{i j}$.

The difference in mass squares of the mass eigenstates in matter can be calculated by diagonalizing Eq. (11) and is shown in Fig. 1 as a function of the matter density for the antineutrinos ${ }^{3}$. Flavour oscillations predominantly occur at the resonance densities, where the effective mass difference between the two relevant mass eigenstates in matter becomes minimum. If the resonances are far apart then each resonance can be treated as an effective two neutrino problem,

\footnotetext{
${ }^{3}$ Since the matter potential $A$ is negative for the antineutrinos, we show the evolution of the mass squares of the mass eigenstates in Fig. 1] as a function of $-\rho$, where $\rho$ is the matter density.
} 
independent of other resonances. For two flavor oscillations the resonance condition is given by

$$
A_{1}=\Delta m^{2} \cos 2 \theta,
$$

where $\Delta m^{2} \equiv m_{2}^{2}-m_{1}^{2}$ is the mass squared difference and $\theta$ is the mixing angle between the two states in vacuum. At the resonance a flip between the mass eigenstates is possible due to the changing mass density. The "flip probability" between the two mass eigenstates is given by

$$
\begin{gathered}
P_{J}=\frac{\exp \left(-\gamma \sin ^{2} \theta\right)-\exp (-\gamma)}{1-\exp (-\gamma)} \\
\gamma=\pi \frac{\Delta m^{2}}{E}\left|\frac{d \ln A_{1}}{d r}\right|_{r=r_{m v a}}^{-1}
\end{gathered}
$$

where $r_{m v a}$ is the position of the maximum violation of adiabaticity (mva) 24] and is defined as

$$
A_{1}\left(r_{m v a}\right)=\Delta m^{2}
$$

For a given set of values of $\Delta m^{2}$ and $\theta$, if the density gradient is small enough so that $\gamma \gg 1$ and $\gamma \sin ^{2} \theta \gg 1$, the flip probability reduces to $P_{J}=\exp \left(-\gamma \sin ^{2} \theta\right) \simeq 0$ and the resonance is called adiabatic. However, if for the given $\Delta m^{2}$ and $\theta$, the density gradient is large such that $\gamma \ll 1$, then $P_{J} \simeq \cos ^{2} \theta$. In that case for very small mixing angles $P_{J} \simeq 1$ and the resonance is completely non-adiabatic. For all intermediate regions $P_{J}$ ranges between $[0-1]$.

For three active neutrinos with their vacuum masses given by the current experimental data and for a static supernova density profile, the resonance condition is satisfied at two distinct widely separated densities inside the supernova [26]. The resonance at the higher density is driven by $\Delta m_{31}^{2}$ and $\theta_{13}$, while that at lower densities is driven mainly by $\Delta m_{21}^{2}$ and $\theta_{12}$. Since the matter induced potential $A_{1}$ is positive (negative) for neutrinos (antineutrinos), the resonance condition given by Eq. (77) is satisfied only if $\Delta m^{2}$ is positive (negative). Since $\Delta m_{21}^{2}$ (for $\left.\theta_{12}<\pi / 4\right)$ is known to be positive at a very high level of confidence from the solar neutrino data, the lower resonance is therefore satisfied only for the neutrino channel. However, $\Delta m_{31}^{2}$ could be either positive (normal hierarchy) or negative (inverted hierarchy) and therefore the higher resonance can occur either in the neutrino or the antineutrino channel. It can be shown that for the most plausible supernova density profiles and the current allowed values of $\Delta m_{21}^{2}$ and $\theta_{12}$, the flip probability at the lower resonance is zero and the transition is completely adiabatic. However, depending on the value of $\theta_{13}$ and the neutrino mass hierarchy, the flip probability at the $\Delta m_{31}^{2}$ driven higher resonance may have any value between [0-1]. We refer readers to [12] for detailed discussion of the three generation oscillation probability in a supernova environment with static density profiles.

Things get more involved when one considers the effect of shock waves on the supernova density profiles. As we will discuss in the following section, the effect of the shock is to cause very sharp jumps in the density gradient. This results in the same $\Delta m^{2}$ producing multiple resonances which are relatively close together. If we assume that the phase effects can be neglected even in this case ${ }^{4}$, then the individual resonances can be considered as independent

\footnotetext{
${ }^{4}$ This is a good approximation especially in our case since we will be working with the IceCube detector which does not have any energy sensitivity. This means that the detected supernova events will be averaged over energy and hence phase effects [25] will be further washed out.
} 
two generation resonances and the net flip probability $P_{H}$ can be expressed in terms of the multiple flip probabilities $P_{i}$ as [26, 18]

$$
\left(\begin{array}{cc}
1-P_{H} & P_{H} \\
P_{H} & 1-P_{H}
\end{array}\right)=\prod_{i=1, n}\left(\begin{array}{cc}
1-P_{i} & P_{i} \\
P_{i} & 1-P_{i}
\end{array}\right)
$$

where $n$ is the number of resonances occurring for the same $\Delta m^{2}$ due to the shock effect.

\subsection{Inclusion of Two Sterile Neutrinos}

For mixing between 3 active neutrinos and 2 sterile neutrinos the form of Eq. (11) remains the same but now we have

$$
U=\prod_{B>A}^{5} \prod_{A=1}^{4} R^{A B}
$$

where $R^{A B}$ is a 5 by 5 rotation matrix about the AB plane,

$$
\begin{gathered}
\mathcal{M}^{2}=\operatorname{Diag}\left(m_{1}^{2}, m_{2}^{2}, m_{3}^{2}, m_{4}^{2}, m_{5}^{2}\right) \\
\mathcal{A}=\operatorname{Diag}\left(A_{1}, 0,0, A_{2}, A_{2}\right) \\
A_{1}= \pm \sqrt{2} G_{F} \rho N_{A} Y_{e} \times 2 E \\
A_{2}= \pm \sqrt{2} G_{F} \rho N_{A}\left(1-Y_{e}\right) \times E
\end{gathered}
$$

where, as in Eq. (4), the "+" and "-" signs in Eq. (15) corresponds to neutrinos and antineutrinos respectively. As before, $i=1-5$ and $\alpha=\mathrm{e}, \mu, \tau, s_{1}$ or $s_{2}$, where $s_{1}$ and $s_{2}$ are two sterile neutrinos. $A_{2}=-A_{N C}$, where $A_{N C}$ is the matter-induced potential of the neutral current interaction of neutrinos/antineutrinos with matter. As discussed in the previous section, all the three active neutrinos/antineutrinos pick up an equal matter-induced potential $A_{N C}$ in matter and the matter induced mass matrix is given by

$$
\begin{aligned}
\mathcal{A}^{\prime} & =\operatorname{Diag}\left(A_{1}+A_{N C}, A_{N C}, A_{N C}, 0,0\right) \\
& =\mathcal{A}+A_{N C} \mathcal{I} .
\end{aligned}
$$

Since the sterile neutrinos do not interact with matter, $A_{N C}$ cannot be completely factored out from the mass squared matrix as in the three neutrino case and hence can be recast as a negative matter potential for the sterile states. The effective mass squared differences between the neutrino/antineutrino eigenstates in matter therefore depend on both $A_{1}$ and $A_{2}$.

The difference in mass squared can again be obtained by diagonalizing the mass squared matrix in matter and the results for the antineutrino channel are shown in Fig. 2 for the different possible mass hierarchies of the five (anti)neutrino system in vacuum. As discussed above, while we know that $\Delta m_{21}^{2}>0$, sign of $\Delta m_{31}^{2}$ is presently unknown. The only experimental information we have on $\Delta m_{41}^{2}$ and $\Delta m_{51}^{2}$ comes from the data of short baseline experiments including LSND and their sign are completely unknown as well. Therefore, in principle its possible to have six 

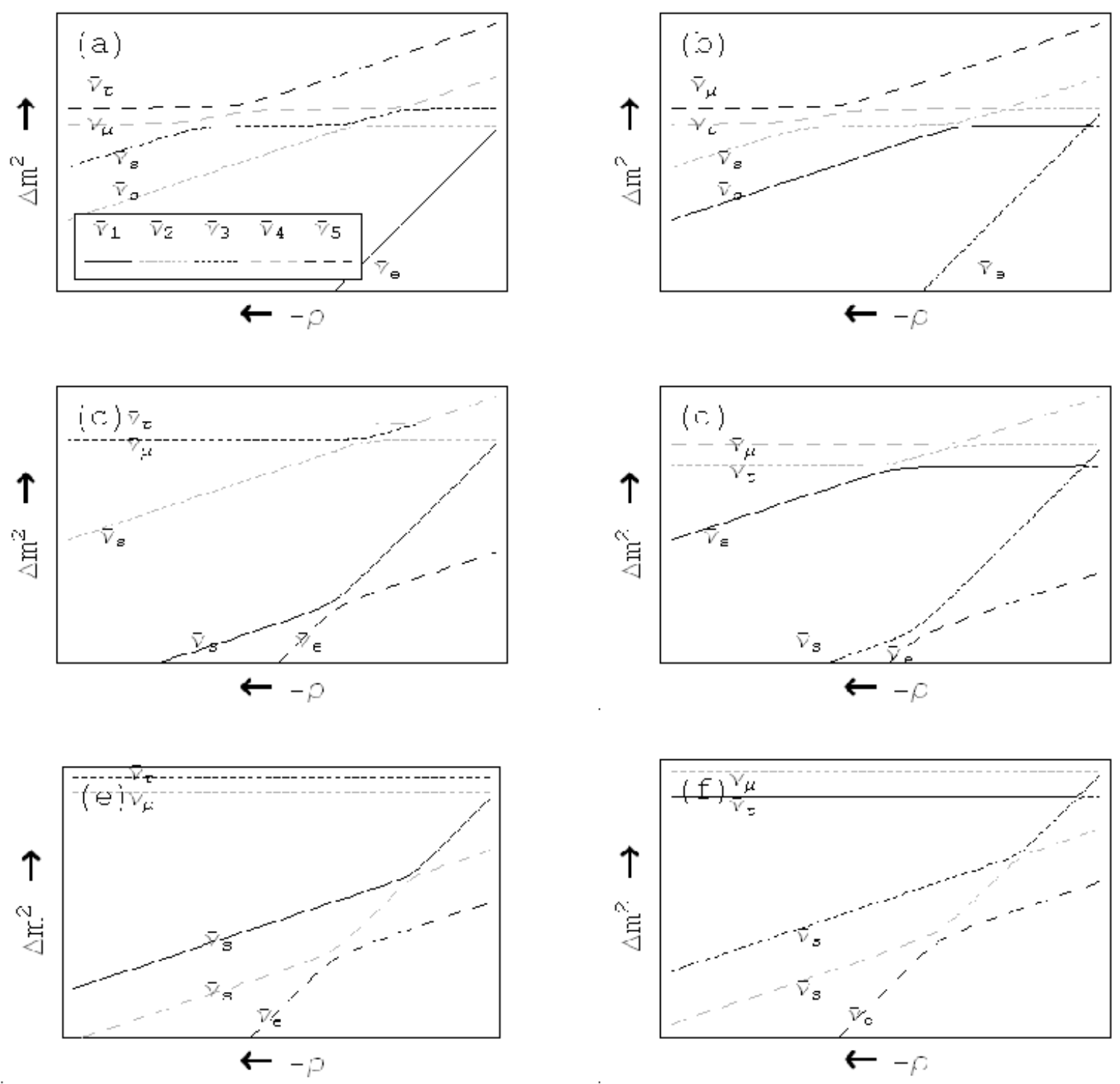

Figure 2: The evolution with density of the mass eigenvalues (squared) for the case of three active anti-neutrinos and two sterile anti-neutrinos, for the mass hierarchies (a) N2+N3, (b) $\mathrm{N} 2+\mathrm{I} 3$, (c) H2+N3, (d) H2+I3, (e) I2+N3 and (f) I2+I3. N3 and I3 correspond to the normal and inverse hierarchies in the active neutrino sector. N2 corresponds to the normal hierarchy in the sterile sector where $\Delta m_{51}^{2}>0$ and $\Delta m_{41}^{2}>0$. H2 corresponds to the half hierarchy in the sterile sector where either $\Delta m_{51}^{2}>0$ and $\Delta m_{41}^{2}<0$, or $\Delta m_{51}^{2}<0$ and $\Delta m_{41}^{2}>0$. I2 corresponds to the inverse hierarchy in the sterile sector where $\Delta m_{51}^{2}<0$ and $\Delta m_{41}^{2}<0$. 
types of mass hierarchy. For simplicity of notation, we will henceforth call them:

(a) N2+N3: $\Delta m_{31}^{2}>0, \Delta m_{41}^{2}>0$ and $\Delta m_{51}^{2}>0$,

(b) N2+I3: $\Delta m_{31}^{2}<0, \Delta m_{41}^{2}>0$ and $\Delta m_{51}^{2}>0$,

(c) H2+N3: $\Delta m_{31}^{2}>0, \Delta m_{41}^{2}>0$ and $\Delta m_{51}^{2}<0$,

(d) H2+I3: $\Delta m_{31}^{2}<0, \Delta m_{41}^{2}>0$ and $\Delta m_{51}^{2}<0$,

(e) I2+N3: $\Delta m_{31}^{2}>0, \Delta m_{41}^{2}<0$ and $\Delta m_{51}^{2}<0$,

(f) I2+I3: $\Delta m_{31}^{2}<0, \Delta m_{41}^{2}<0$ and $\Delta m_{51}^{2}<0$.

As discussed in the previous section the passage of the shock wave causes each $\Delta m^{2}$ to have multiple resonances. The additional resonances due to the inclusion of sterile neutrinos can also be considered as independent, and the net flip probability is given by equation [1],

\subsection{Experimental Bounds}

Here we very briefly review the current knowledge we have about the masses and mixing angles of the active and sterile neutrinos from all available neutrino oscillation data.

\subsubsection{Solar Data}

The combined analysis of world data on solar neutrinos 3 and the KamLAND reactor data 4 has established the so-called Large Mixing Angle (LMA) solution as the solution to the solar neutrino anomaly with the current best-fit parameters [27] $\Delta m_{\odot}^{2} \equiv \Delta m_{21}^{2}=8.0 \times 10^{-5} \mathrm{eV}^{2}$ and $\sin ^{2} \theta_{\odot} \equiv \sin ^{2} \theta_{12}=0.31$ and the $3 \sigma$ allowed range given by [27, 28]

$$
\begin{aligned}
0.25 & <\sin ^{2} \theta_{12} \\
7.2 \times 10^{-5} \mathrm{eV}^{2} & <\Delta m_{21}^{2}<9.2 \times 10^{-5} \mathrm{eV}^{2}
\end{aligned}
$$

\subsubsection{Atmospheric Data}

The zenith angle dependent event spectrum of the atmospheric neutrino data from SuperKamiokande [5] and the data from the long baseline K2K experiment [6] can be best explained in

terms of almost pure $\nu_{\mu}-\nu_{\tau}$ oscillations with best-fit parameters $\Delta m_{\text {atm }}^{2} \equiv\left|\Delta m_{31}^{2}\right|=2.1 \times 10^{-3}$ $\mathrm{eV}^{2}$ and $\sin ^{2} 2 \theta_{\mathrm{atm}} \equiv \sin ^{2} 2 \theta_{23}=1$ and the $3 \sigma$ allowed range given by [5]

$$
\begin{array}{ccl}
\sin ^{2} 2 \theta_{23} & > & 0.9 \\
1.3 \times 10^{-3} \mathrm{eV}^{2}< & \Delta m_{31}^{2} & <4.2 \times 10^{-3} \mathrm{eV}^{2}
\end{array}
$$

\subsubsection{CHOOZ Reactor Data}

The upper limit on the mixing angle $\theta_{13}$ is mainly determined by the reactor neutrino experiments CHOOZ and Palo Verde [7]. Data from these experiments when combined with the solar and atmospheric neutrino data gives at $3 \sigma$ the bound [28, 29].

$$
\sin ^{2} \theta_{13}<0.044
$$




\begin{tabular}{|c|c|c|}
\hline Parameter & Best fit & Best fit in sub-eV \\
\hline$\Delta m_{41}^{2}$ & $0.92 \mathrm{eV}^{2}$ & $0.46 \mathrm{eV}^{2}$ \\
$\Delta m_{51}^{2}$ & $22 \mathrm{eV}^{2}$ & $0.89 \mathrm{eV}^{2}$ \\
$U_{e 4}$ & 0.121 & 0.090 \\
$U_{\mu 4}$ & 0.204 & 0.226 \\
$U_{e 5}$ & 0.036 & 0.125 \\
$U_{\mu 5}$ & 0.224 & 0.160 \\
\hline
\end{tabular}

Table 1: The two best fit solutions for the short baseline experiments taken from [10].

\subsubsection{Data from Short Baseline Experiments}

A combined analysis of Bugey, CHOOZ, CCFR84, CDHS, KARMEN, NOMAD, and LSND for the case of a 3+2 mass hierarchy gives two possible solutions [10]. These are shown in Table 1] Column 2 of Table 1 shows the global best-fit, while column 3 gives the solution if all neutrino masses were restricted to lie in the sub-eV range ${ }^{5}$. Note that the values of $\theta_{34}, \theta_{35}$ and $\theta_{45}$ are not constrained by any experiments to date. However in what follows, we shall assume that all concerned mixing angles (including $\theta_{13}$ ) are large enough so that all the transition probabilities are adiabatic for the static density profile of the supernova in absence of shock effects. We shall briefly discuss in section 4.4 the effect of reducing the mixing angles and hence the degree of adiabaticity on the resultant supernova neutrino signal.

\section{Supernova Neutrinos and Shock waves}

\subsection{Time Dependent Luminosity and Energy Spectra.}

About $3 \times 10^{53}$ ergs of energy is released in a type-II supernova, $99 \%$ of which is in the form of neutrinos. These neutrinos drift out from the dense core of the proto-neutron star and beyond a certain radius determined by the energy of the neutrino, travel freely and escape. This radius of last scattering which characterizes the energy distribution of the neutrinos is generally called the "neutrinosphere". Neutrinos are mainly released in two phases. A short "neutrinonization burst" of pure $\nu_{e}$ is produced by electron capture on protons when shock wave crosses the neutrinosphere. Subsequently the majority of the neutrinos are produced as $\nu-\bar{\nu}$ pairs of all three flavours over a period of 10-20 seconds as the proto-neutron star cools.

Even if all the six species of neutrinos are dominantly produced by the same mechanism, their luminosities and energies at their respective neutrinospheres can differ due the difference in their degree of interaction with matter. If the effect of weak magnetism and muon production inside the supernova core is neglected, the spectra of $\nu_{\mu}, \bar{\nu}_{\mu} \nu_{\tau}$ and $\bar{\nu}_{\tau}$ are approximately equal, and so they are often grouped together and collectively denoted as $\nu_{x}$. An accurate prediction of the time dependent energy spectra of the emitted neutrinos requires a full simulation including all significant neutrino interactions. To date only the Lawrence Livermore group 22 has published detailed results of the energy spectra of the neutrinos over the full duration of the supernova, in

\footnotetext{
${ }^{5}$ Note that for the best-fit solution shown in column 2 , the sum of neutrino masses are in conflict with the current cosmological bounds (see for instance [30] and reference therein). However, we still use them in this paper as an illustrative example for the case where the sterile neutrinos are non-degenerate.
} 
a simulation with a successful explosion. The resulting luminosity and average energies of the neutrinos taken from 22, are shown in Fig. 3. It may be seen that the luminosities of all the six types of neutrinos are very similar (apart from the $\nu_{e}$ neutronization burst), but the average energies of $\nu_{x}$ are considerably larger than that of $\nu_{e}$ and $\bar{\nu}_{e}$ throughout the duration of the supernova. Since neutrino oscillations effectively flip the energy spectra of the $\nu_{e}$ and/or $\bar{\nu}_{e}$ with that of the $\nu_{x}$ and since the extent of this flip depends on the value of the mixing angle and the neutrino mass hierarchy, this difference in the initial energy spectra of the different (anti)neutrino species has been used in previous studies in the context of three [12] and four [13] neutrino oscillations to put bounds on the neutrino mass spectrum and mixing angles by exploiting the energy dependence of the cross sections relevant to the detectors on earth. However, more recent work [23] on neutrino transport inside the supernova has cast doubt on the magnitude of this effect. When all neutrino scattering processes, some of which were not included in the Livermore study, are included, the average energies of the different (anti)neutrino flavors, particularly $\bar{\nu}_{e}$ and $\nu_{x}$ become very similar. The condition of equipartition of luminosity between the different species also breaks down and in some simulations one might find the $\bar{\nu}_{e}$ luminosity to be almost double that of $\nu_{x}$.

In what follows, we shall use the time dependence of the luminosity as given by the Livermore group in our estimates of neutrino oscillation effects as this should not be so sensitive to the additional scattering processes not included in the Livermore analysis. However when considering the oscillations among the three active neutrinos we will estimate the uncertainties by computing the expected signal both for the Livermore luminosities and energy distributions and for the case where the energy distributions are equal but the luminosities differ in the manner just discussed. For the case of oscillation into sterile neutrinos the results are relatively insensitive to these differences as the signal does not depend on a difference between $\bar{\nu}_{x}$ and $\bar{\nu}_{e}$.

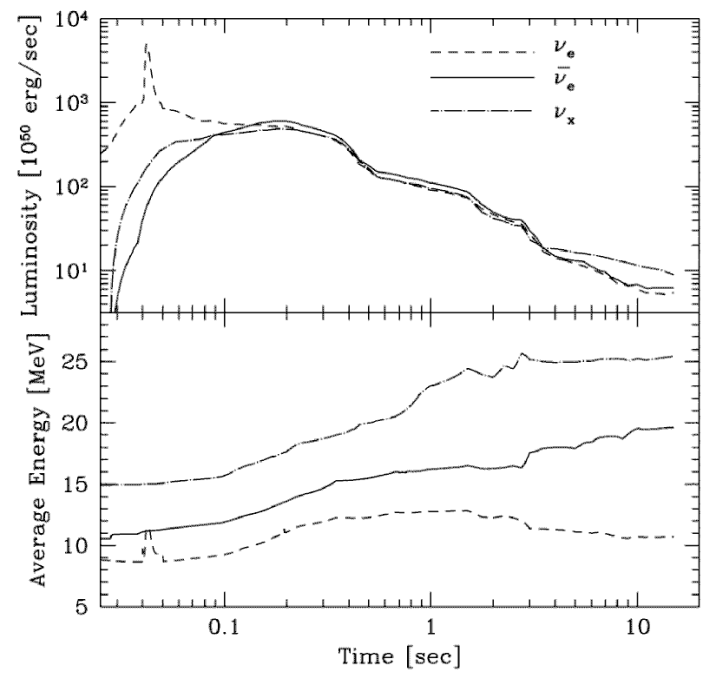

Figure 3: The time evolution of the luminosity and average energy as calculated by the Lawrence Livermore group. 


\subsection{Supernovae Shock Wave(s)}

In this analysis we are particularly interested in the shock waves that form within the supernovae. It is believed that when the core of the collapsing star reaches nuclear density, the collapse rebounds forming a strong outward shock. This is stalled and then regenerated by a neutrino driven wind. During this process both a forward and a reverse shock may form [15. In order to get a realistic estimate of the density profile of the shock wave it is necessary to use results from numerical simulations. However, since detailed numerical results from such simulations are not available to us, we will use a simplified profile for the shockwave used in [18, 20]. In order to exhibit the impact of both the forward and reverse shock on the resultant neutrino signal in earth-bound detectors, we will consider scenarios where we (i) neglect the effect of the shock wave, (ii) consider the effect of the forward shock alone and (iii) consider both the forward and reverse shock in calculating the oscillation probabilities. The snap shot of the density profile

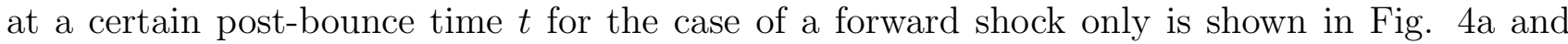
the case of both a forward and reverse shock is shown in Fig. 5 5 . The shape of the shockwave remains roughly constant in time but the height varies as the shockwave propagates through the supernovae (c.f.Fig 1 [18). The effect of the shock wave on the neutrino oscillation probabilities has been discussed before in [14, 18, for the forward shock and in [15, 20] for the forward and reverse shock. We will briefly review it again in the next subsections for completeness.
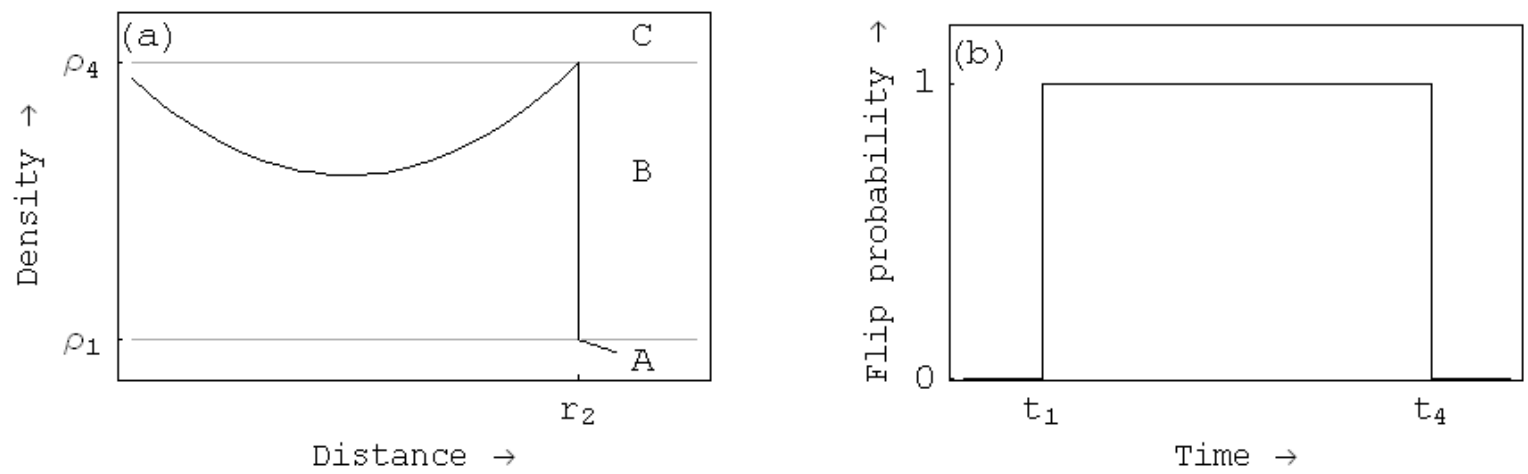

Figure 4: (a) The density profile of the forward shock as a function of the distance from the core of the supernova. (b) The resulting flip probability as a function of time.

\subsection{Effect of the Shock Wave}

\subsubsection{Forward Shock Only}

We will consider here the effect of the shockwave on the neutrinos emerging from the supernova in the case where all the relevant mixing angles are sufficiently large so that the resonance for the static density profile in the absence of the shockwave is completely adiabatic.

The density structure shown in Fig. 榲 exhibits three separate regions. Initially, for times $t<t_{1}$, the resonant density $\rho_{R}\left(=\left(\Delta m^{2} \cos 2 \theta\right) /\left(2 \sqrt{2} G_{F} N_{A} Y_{e} E\right)\right)$ lies below the density at which the shock wave appears, $\rho_{R}<\rho_{1}$, i.e. in region A. The resonance crossing is adiabatic to a good approximation in this region and therefore the flip probability vanishes for $t<t_{1}$ 

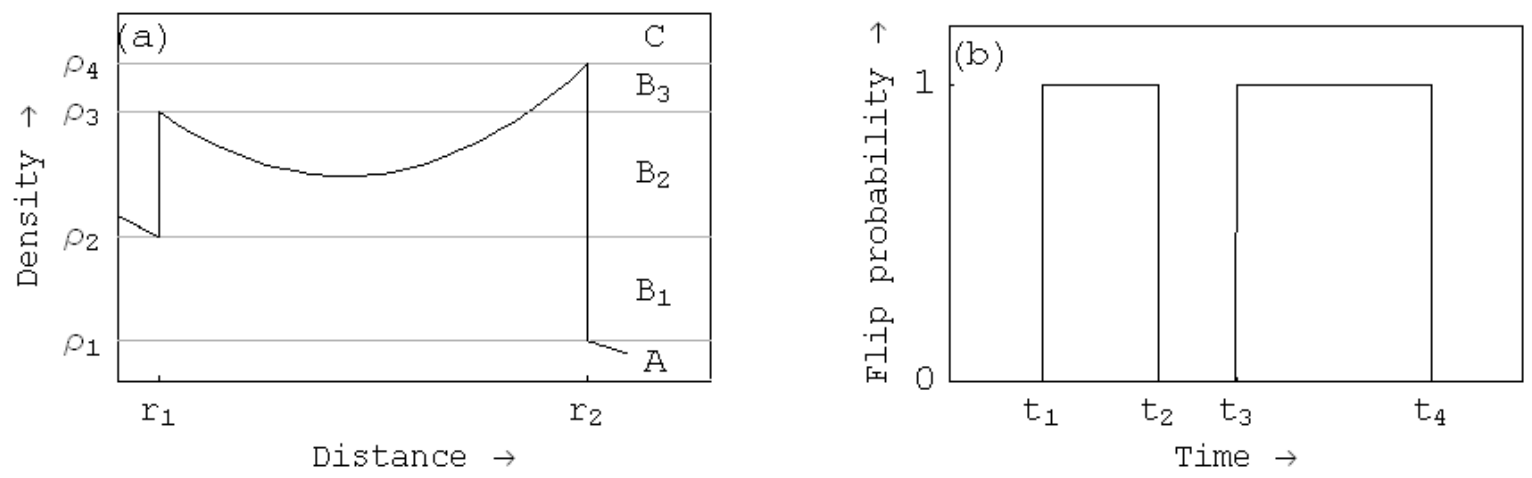

Figure 5: (a) The density profile of the forward and reverse shock as a function of the distance from the core of the supernova. (b) The resulting flip probability as a function of time.

as shown in Fig. 40. As the shockwave moves out $\rho_{1}$ decreases and for times $t_{1}<t<t_{4}$ the resonant density lies in region $B, \rho_{1}<\rho_{R}<\rho_{4}$. Due to the large density gradient at the shock front, in this region the flip probability increases to $\cos ^{2} \theta \simeq 1$ (c.f. eq(8)) and therefore the resonance becomes non-adiabatic in the period $t_{1}<t<t_{4}$ as shown in Fig. 4b. As may be seen from the figure, the neutrino pass through two adiabatic resonances and one non-adiabatic resonance. The flip probability passing through each adiabatic resonances is approximately zero and therefore the total flip probability is equal to the flip probability passing through the non-adiabatic resonance alone. Finally in region $\mathrm{C}$ the shockwave has passed through the resonant density so that $\rho_{4}<\rho_{R}$ and the resonance is adiabatic, therefore the flip probability is approximately zero again for $t>t_{4}$.

\subsubsection{Forward and Reverse Shock}

The situation is illustrated in Fig 5. For the density in the appropriate range there will now be two non-adiabatic resonances, corresponding to the points $r_{1}$ and $r_{2}$. As before at early times $t<t_{1}$, the resonant density $\rho_{R}$ lies below the density at which the shock wave appears, $\rho_{R}<\rho_{1}$, i.e. in region $A$. As before, to a good approximation in this region the resonance crossing is adiabatic and so the flip probability vanishes for $t<t_{1}$ as is shown in Fig. 5]. As the shockwave moves out $\rho_{1}$ decreases and for times $t_{1}<t<t_{2}$ the resonant density lies in region $B_{1}$ such that $\rho_{1}<\rho_{R}<\rho_{2}$. Thus in region $B_{1}$ only the forward shock has reached the resonant density and is therefore equivalent to region $\mathrm{B}$ in the forward shock only case. At later times $t_{2}<t<t_{3}$ the resonant density lies in region $B_{2}$ where $\rho_{2}<\rho_{R}<\rho_{3}$. In this region the neutrino passes through the resonant density at both the forward and reverse shock and both are non-adiabatic resonances. Therefore the mass eigenstates are flipped at the reverse shock and then flipped back at the forward shock. When the mixing angle is non-zero the flip probability at the forward and reverse shocks is $\cos ^{2} \theta$ and the total flip probability is $\frac{1}{2} \sin ^{2} 2 \theta$. Between $t_{3}<t<t_{4}$, the shock profile corresponds to region $B_{3}$ where $\rho_{3}<\rho_{R}<\rho_{4}$ and the neutrinos cross their resonance density through the forward shock only. Hence the flip probability again increases suddenly to $\cos ^{2} \theta$. For $t>t_{4}$, we are in region $C$ where $\rho_{4}<\rho_{R}$ and the shockwave has passed through the resonant density. The resonance is therefore again adiabatic. The total flip probability of the 


\begin{tabular}{|c|c|c|c|c|c|}
\hline Model & $\left\langle E_{\nu_{e}}^{0}\right\rangle$ & $\left\langle E_{\bar{\nu}_{e}}^{0}\right\rangle$ & $\left\langle E_{\nu_{x}}^{0}\right\rangle$ & $\frac{\Phi_{\nu_{e}}^{0}}{\Phi_{\nu_{x}}^{0}}$ & $\frac{\Phi_{\bar{\nu}_{e}}^{0}}{\Phi_{\nu_{x}}^{0}}$ \\
\hline LL & 12 & 15 & 24 & 2.0 & 1.6 \\
G1 & 12 & 15 & 18 & 0.8 & 0.8 \\
G2 & 12 & 15 & 15 & 0.5 & 0.5 \\
\hline
\end{tabular}

Table 2: The average energies and total fluxes characterising the primary neutrino spectra produced inside the supernova. The numbers obtained in the Lawrence Livermore simulations are denoted as LL, while those obtained by the Garching group are denoted as G1 and G2.

neutrinos for the case where we have both forward and reverse shock has the form of a double peak as is shown in Fig. 5. The same structure of the flip probability can be caused by a density profile where the two non-adiabatic resonances which are not "overlapping", such as Fig. 6, where the flip probability is only non-zero in regions $D_{1}$ and $D_{3}$. However this also requires that there exists a forward and reverse shock.

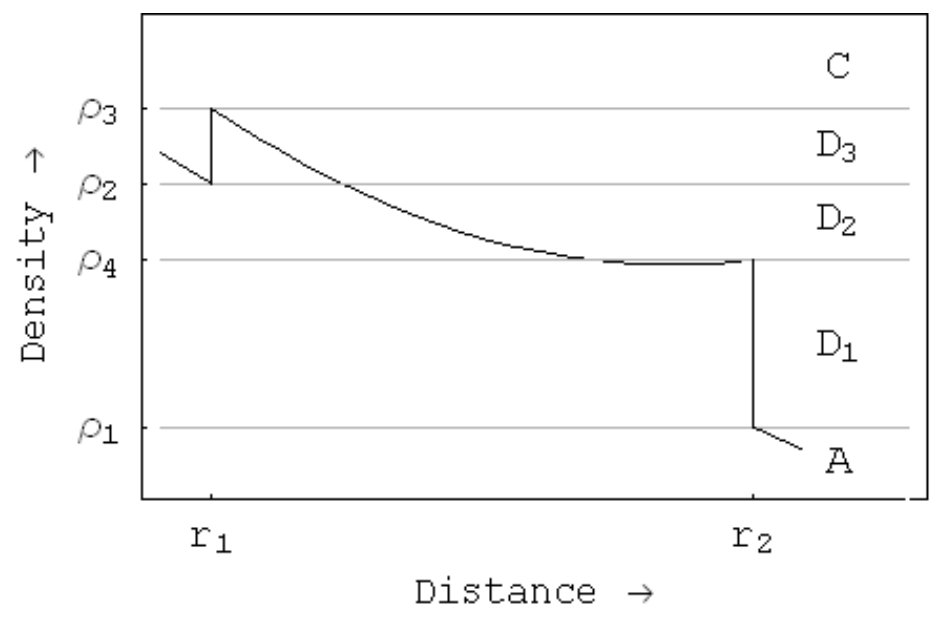

Figure 6: An alternate density profile giving the same flip probability as shown in figure 5 .

\section{Probing Supernovae Neutrino Oscillations in IceCube}

In this Section we present the neutrino induced signal expected in the IceCube detector for a galactic supernova event. We first give the results for the case of three active neutrinos and then take up the case where two additional sterile neutrinos are added to the neutrino mass spectrum. We first turn to the discussion of the properties of the IceCube detector.

\subsection{IceCube as a Supernova Detector}

IceCube is a future $\mathrm{km}^{3}$ neutrino telescope, under construction in Antartica [17]. On completion, it will contain 4800 optical modules deployed into the ice which will detect the Cerenkov photons. Though designed to observe ultra high energy neutrinos, IceCube can detect a supernova through 
its neutrinos by detecting Cerenkov light from $e^{+}$produced by $\bar{\nu}_{e}$ capture on protons. Each event cannot be distinguished in the detector, but the addition to the background photon halo coming from the Cerenkov radiation associated with the produced positron can be measured. The number of additional photons is

$$
N_{d e t}=\frac{47.75}{M e V m^{-3}} f_{C h} f_{a b s} f_{O M} \rho \frac{1}{4 \pi D^{2}} \int_{t_{\min }}^{t_{\max }} d t \int_{0}^{\infty} d E \sigma(E) E F(E, t)
$$

where $f_{C h}, f_{a b s}$ and $f_{O M}$ are fudge factors of order 1 and are given in the appendix, $\rho=6.18 \times 10^{25}$ $\mathrm{m}^{-3}$ is the density of targets in ice, $\sigma\left(\right.$ in $\left.\mathrm{cm}^{2}\right)$ is the cross section for $\bar{\nu}_{e} p \rightarrow n e^{+}, E$ (in MeV) is energy, $\mathrm{D}\left(\right.$ in $\mathrm{cm}^{2}$ ) is the distance to the supernova, $\mathrm{t}$ (in $\mathrm{s}$ ) is time, $\mathrm{F}$ (in $\mathrm{MeV}^{-1} \mathrm{~s}^{-1}$ ) is the flux of anti-electron neutrinos. The initial spectra of neutrino species $\nu_{\alpha}$ from a supernova is parameterized as 31 ]

$$
F^{0}(E, t)=\frac{\Phi(t)}{\langle E\rangle(t)} \frac{(\alpha(t)+1)^{\alpha(t)+1}}{\Gamma(\alpha(t)+1)}\left(\frac{E}{\langle E\rangle(t)}\right)^{\alpha(t)} \exp \left(-(\alpha(t)+1) \frac{E}{\langle E\rangle(t)}\right)
$$

where $\langle E\rangle$ and $\Phi$ are the average energy and total number flux and $\alpha$ is a dimensionless parameter which typically takes the values 2.5-5. For the results presented in this paper, we have assumed $\alpha_{\bar{e}}=3$ and $\alpha_{x}=4$. In order to compare the impact of the uncertainties on the average energies and fluxes of the neutrinos obtained in different supernova computer simulations, we will present our results using supernova neutrino parameters given by both the Lawrence Livermore and Garching groups. Specifically, we consider three cases shown in Table 2 [15].

The neutrino flux in the detector is

$$
F_{\beta}=\sum_{\alpha} F_{\alpha}^{0} P_{\alpha \beta}
$$

where

$$
\begin{gathered}
P_{\alpha \beta}=\sum_{i} P_{\alpha i}^{m} P_{i \beta}^{\oplus} \\
P_{\alpha i}^{m}=\sum_{j}\left|U_{\alpha j}^{m}\right|^{2} P_{i j} \\
P_{i j}=\left|\left\langle\nu_{i} \mid \nu_{j}^{m}\right\rangle\right|^{2}
\end{gathered}
$$

$P_{\alpha i}^{m}$ is the probability that a $\left|\nu_{\alpha}\right\rangle$ produced inside the supernova emerges in the $i^{\text {th }}$ mass eigenstate, $\left|\nu_{i}\right\rangle . U_{\alpha j}^{m}$ is the mixing matrix at the point of production, the neutrinosphere. $P_{i j}$ is the probability that a $\left|\nu_{j}^{m}\right\rangle$ mass eigenstate in matter appears as $\left|\nu_{i}\right\rangle$ mass eigenstate in a vacuum, this is known as the flip probability. $P_{i \beta}^{\oplus}$ is the probability of detecting the $\left|\nu_{i}\right\rangle$ mass eigenstate in the $\left|\nu_{\beta}\right\rangle$ weak interaction eigenstate. In this paper, we do not take into account Earth-matter effects and therefore

$$
P_{i \beta}^{\oplus}=\left|U_{i \beta}\right|^{2}
$$




\subsection{IceCube Background Signal}

IceCube has an irreducible background photon halo with energies comparable to those produced by the supernova neutrinos. In the analysis of a supernova signal the additional number of photons need to be distinguished from this background. The mean number of detected photons is

$$
\bar{n}_{i}=N_{M} \nu_{1 p e} t_{b i n}+N_{\text {det }}
$$

where $N_{M}$ is the number of optical modules, $\nu_{1 p e}$ is the mean background detection rate per optical module, $t_{b i n}$ is length of the time bin and $N_{\text {det }}$ is the signal. Using Poisson statistics the fluctuations in the background photon halo is given by $\sqrt{n_{i}}$. The background rate is $500 \mathrm{~Hz}$ per optical module and IceCube would have 4800 optical modules. Thus for time bin of $100 \mathrm{~ms}$ the random fluctuations are expected to be $\sigma=\sqrt{2.4 \times 10^{5}+N_{\text {det }}}$.

In addition there is an error due to the uncertainty in the time resolution, $\delta t$, of the detector giving

$$
\begin{aligned}
\Delta n_{i}^{+} & =\frac{\delta t}{t_{b i n}}\left(n_{i+1}+n_{i-1}\right) \\
\Delta n_{i}^{-} & =\frac{\delta t}{t_{b i n}} 2 n_{i} .
\end{aligned}
$$

where $t_{b i n}$ is the size of each time bin and we take a detector time resolution of $\delta t=10 \mathrm{nsec}$ for our estimate of the uncertainties. In our analysis we will use as an upper and lower estimate of the true event number the values $n_{i}+\Delta n_{i}^{+}+\sigma$ and $n_{i}-\Delta n_{i}^{-}-\sigma$ respectively, where $n_{i}$ is the number detected in the $i^{\text {th }}$ bin. In our numerical plots we will show only the additional photons produced by the supernova neutrinos, but for the fluctuations we will include all errors as discussed above.

\subsection{Results}

\subsubsection{Three Active Neutrinos}

We first consider the case of a "standard" supernova at $10 \mathrm{kpc}$ from earth with average energy and luminosity for the neutrinos taken from the Lawrence Livermore group simulation. The initial spectrum of neutrinos used is that given in Eq. (24). At the neutrinosphere the matterinduced potential is much greater than the vacuum mass squared differences; for anti-neutrinos this is large and negative. Therefore $\bar{\nu}_{e}$ is approximately equal to the mass eigenstate with the lowest mass squared. In the normal hierarchy this is $\bar{\nu}_{1}$ and in the inverted hierarchy this is $\bar{\nu}_{3}$. Fig. 1 1shows the evolution with density of the mass squared of the anti-neutrino mass eigenstates for the normal and inverted hierarchies. From this one may readily see if there is a level crossing and determine the $\bar{\nu}_{e}$ flux in the detector using Eqs. (25) and (26). The relevant expressions for the probabilities are given in Table 3. The resonant oscillation effects for anti-neutrinos occur only if there is an inverted mass hierarchy. In this case the flux of electron anti neutrinos in the detector is given by

$$
F_{e}=F_{x}^{0}+P_{e e}\left(F_{e}^{0}-F_{x}^{0}\right)
$$

where

$$
P_{e e}=P_{13}\left|U_{e 1}\right|^{2}+\left(1-P_{13}\right)\left|U_{e 3}\right|^{2}
$$




\begin{tabular}{|c|c|c|c|}
\hline Mass hierarchy & $\mathrm{i}$ & $P_{e i}^{m}$ & $P_{x i}^{m}$ \\
\hline \hline Normal & 1 & 1 & 0 \\
& 2 & 0 & 1 \\
& 3 & 0 & 1 \\
\hline Inverted & 1 & $P_{13}$ & $1-P_{13}$ \\
& 2 & 0 & 1 \\
& 3 & $1-P_{13}$ & $P_{13}$ \\
\hline
\end{tabular}

Table 3: The probabilities $P_{e i}^{m}$ and $P_{x i}^{m}$ for three active neutrinos, where $P_{\alpha i}^{m}$ is given by Eq. (26) and $P_{i j}$ is the flip probability at the resonance between the $\nu_{i}$ and $\nu_{j}$ mass eigenstates. Only $P_{13}$ appears in the expression for the probabilities since $P_{12}=0$ for the current values of $\Delta m_{21}^{2}$ and $\sin ^{2} \theta_{12}$.

From Eq. (33) we see that if the initial flux $F_{\alpha}^{0}(E)$ of the $\bar{\nu}_{e}$ and $\bar{\nu}_{x}$ are identical, there will be no observable oscillation effect. In the LL case the average energy of the $\nu_{x}$ is much larger than that of the $\bar{\nu}_{e}$. Since the detection cross-section increases quadratically with anti-neutrino energy, flavor oscillations inside the supernova are expected to enhance the anti-neutrino signal in IceCube. In Fig. 7 the number of photons that would be detected by IceCube due to the supernova anti-neutrinos is shown for the normal hierarchy and for the inverted hierarchy for the case of no shock wave, a forward shock wave and a forward and reverse shock. In the figure the width of the line represents the upper and lower event number as discussed in the previous Section. Thus signals much larger than this width should be observable.

Due to the energy dependence of the interaction cross section in the detector the more energetic component will give the larger signal. In the case of a normal hierarchy there are no resonant level crossings for the antineutrinos and the $\bar{\nu}_{e}$ flux on earth originated as the $\bar{\nu}_{e}$ flux at the neutrinosphere (c.f. Table 3). Since in this case the resultant neutrino flux has the least energetic component, it explains why the normal hierarchy case in Fig. 7 corresponds to the lowest bound for the number of photons in the detector at all times. For inverted hierarchy if we ignore the effect of shock waves, then for large values of $\theta_{13}$ the transition is completely adiabatic and the resultant $\bar{\nu}_{e}$ flux on earth originated as the $\bar{\nu}_{x}$ flux at the neutrinosphere. Since in this case the $\bar{\nu}_{e}$ flux at the detector is made up entirely of the most energetic component, the resulting number of photons provide the upper bound on the expected number of photons in the detector. The effect of the shock wave is to bring about abrupt changes in the oscillation probability as discussed in the Section 3.3.2 In particular, we had seen that the transition probability flips from being completely adiabatic to almost non-adiabatic at the shock front. Therefore, including the effect of the shock wave means that the $\bar{\nu}_{e}$ flux on earth at the time when the shock front crosses the resonance region originated as the $\bar{\nu}_{e}$ flux at the neutrinosphere, causing the reduction seen in the signal at these times in Fig. 17. We see that the structure in the signal for the forward and reverse shock does show a single and double bump structure respectively, conforming to the shape of the oscillation probability discussed in Section 3.3 .2

In both the single and double shock cases the structure in the signal above the photon background is clearly visible, demonstrating that IceCube will indeed be capable of distinguishing between the normal and inverted hierarchy for the case the initial energy spectra of different flavours differ. However, as discussed in Section 3.1 the inclusion of further scattering processes within the supernovae is expected to have the effect of reducing the difference in the average 

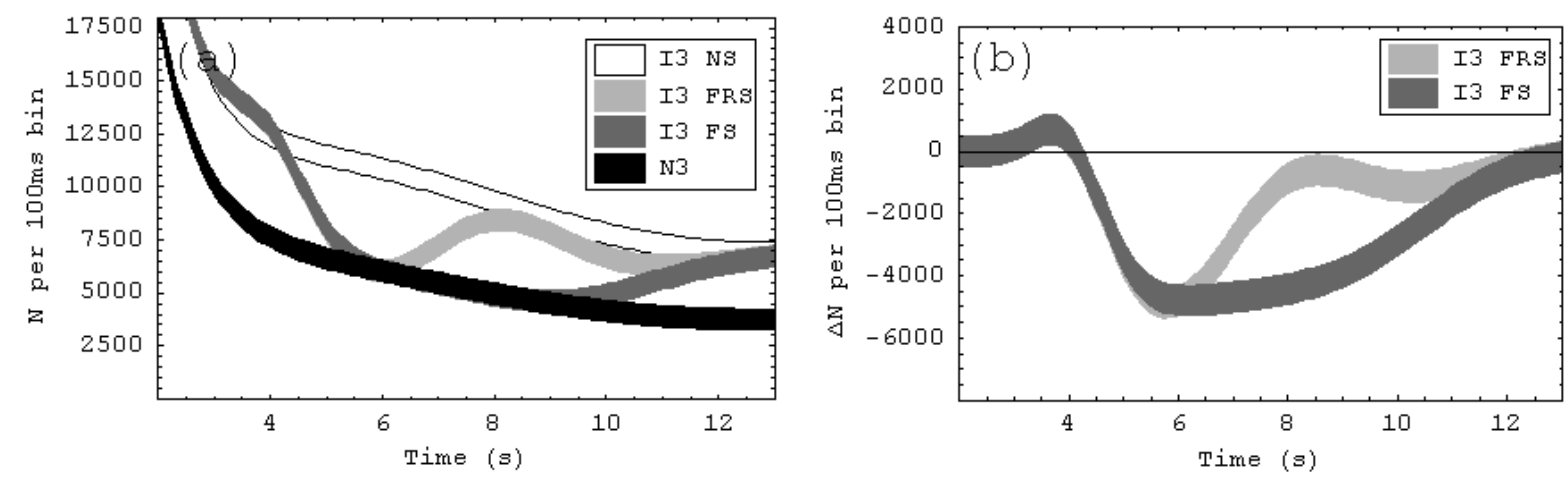

Figure 7: The left hand panel shows the number of photons $(N)$ that would be detected in IceCube for the case of three active neutrinos only with either normal or inverted hierarchy. For inverted hierarchy we show the results where we disregard the shock effect (NS), consider only the effect of the forward shock (FS) and take effect of both the forward and reverse shocks (FRS). For normal hierarchy, the shock does not have any effect and we have the results denoted as N3 in the figure for all cases. The right hand panel shows the difference $(\Delta N)$ between the number of photons expected in presence of shock to the number of photons in absence of shock effects. The observed photons are split into 100ms time bins and the width of the lines reflect the expected fluctuation in the photons detected.

energy between the $\bar{\nu}_{e}$ and $\bar{\nu}_{x}$ components. In this case the structures in the signal just discussed and indeed the effect of oscillations itself due to the energy differences between the different (anti)neutrino flavors will not be present. However all is not lost because the effect of the additional scattering processes, while reducing the energy difference, increases the difference in number flux of the neutrinos with the number flux of the $\bar{\nu}_{e}$ becoming almost twice that of the $\bar{\nu}_{x}$ components during the accretion phase (cf. Table 21). To make this quantitative we have calculated the number of photons expected in the IceCube detector for the cases G1 and G2 of Table 2. Since results for the Garching simulations are not available over the full time period of the supernova, we have used the average energy and total fluxes for G1 and G2 from Table 2 but the time dependent fall of the flux from the simulations of LL. The results are presented in Fig. 8, where the upper panels are for the case G1 and lower ones for G2. The results we get are very similar to the one we had for the LL case. The reason is that even though the ratio of $\left\langle E_{\bar{\nu}_{e}}^{0}\right\rangle /\left\langle E_{\nu_{x}}^{0}\right\rangle$ is nearly 1 for the Garching simulations, the ratio of the fluxes $\Phi_{\bar{\nu}_{e}}^{0} / \Phi_{\nu_{x}}^{0}$ is nearly half. This means that in this case even though neutrino oscillation does not change the energy of the resultant $\bar{\nu}_{e}$ spectra emerging from the supernova, it changes the total number flux, increasing the number of $\bar{\nu}_{e}$ and hence producing an enhanced signal in the detector.

Note that the mere existence of structure due to the shock waves provides the evidence for the inverted hierarchy. However as is evident from Figs. 7 and 8 the signal contains much more information, the sign of the effect giving information about the relative importance of an energy difference or luminosity difference between the $\bar{\nu}_{e}$ and $\bar{\nu}_{x}$ components. Also the shape of the signal provides seismological information capable of determining much about the nature of the supernova shock wave or waves. Clearly, given the sensitivity of the signal to the 

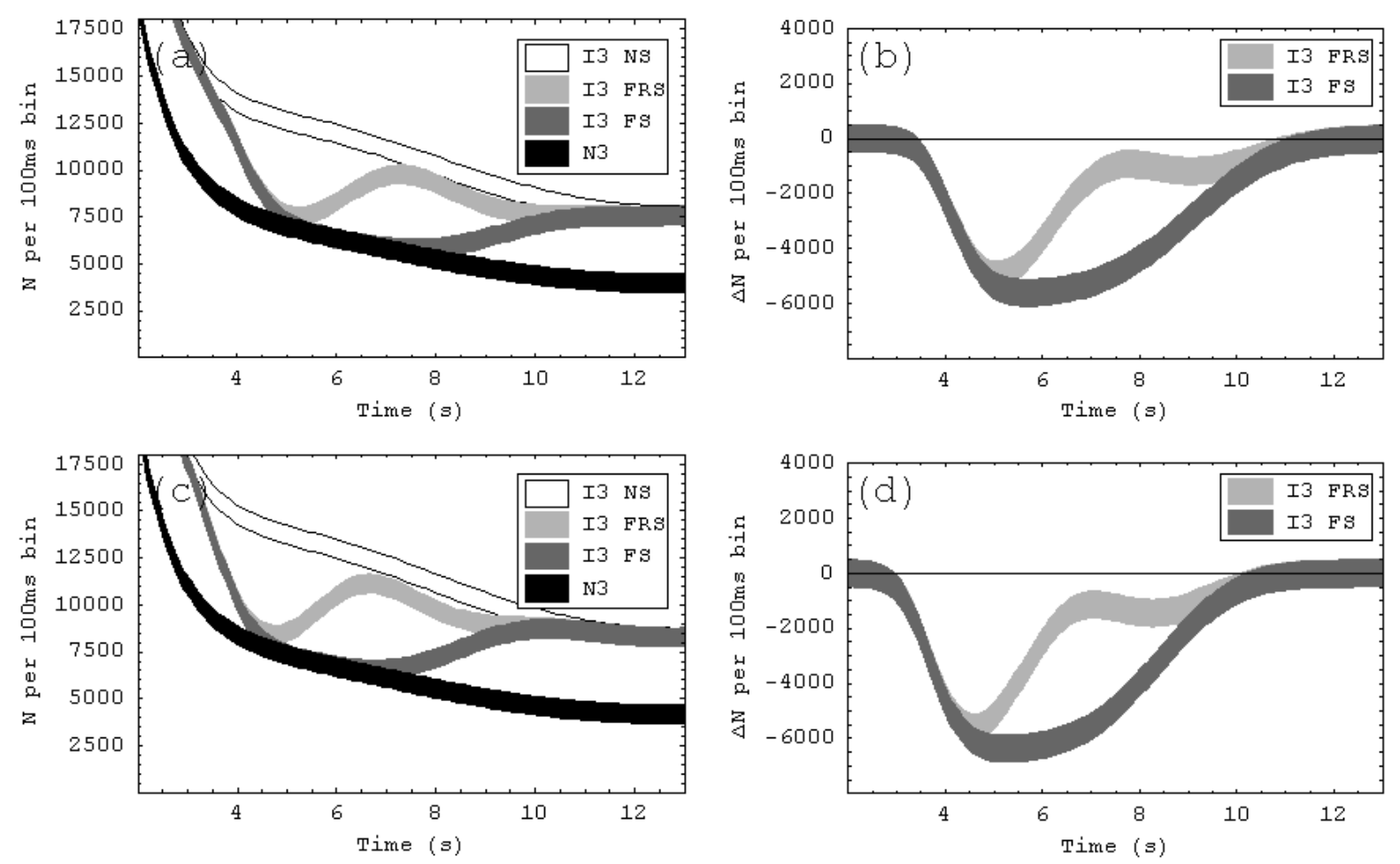

Figure 8: Same as Fig. [7 but with equal initial energy spectra for the active antineutrinos and different luminosities as discussed in the text. (a) and (b) are for the case called G1 while (c) and (d) show the case G2.

initial spectra and luminosities, it is desirable to have a full time dependent simulation of the luminosity and energy spectrum of the Lawrence Livermore type but including all significant neutrino interactions within the neutrinosphere.

\subsubsection{Three Active and Two Sterile Neutrinos}

We turn now to the case in which there are additional sterile neutrinos capable of explaining the LSND anomaly. Once again resonant effects due to the passage of shock waves through the supernova can give significant information about the neutrino mixing. In this case we do not have to rely on a detailed knowledge of the luminosities and energy spectra of the active neutrinos because oscillation to a sterile neutrino necessarily corresponds to a reduction in the signal and therefore it's much easier to observe oscillation and shock effects in this case.

The analysis proceeds in a manner similar to that for the three neutrino case. We start with the evolution of the mass squared of the mass eigenstates for the different possible hierarchies as shown in Fig. 2. From this it is straightforward to compute the $\bar{\nu}_{e}$ flux in the detector using Eqs. (25) and (26). Table 4 gives the probabilities $P_{e i}^{m}$ and $P_{x i}^{m}$ for the different neutrino mass spectra. This simplifies for the case considered here, $\left|U_{e 1}\right|^{2},\left|U_{e 2}\right|^{2}>>\left|U_{e 3}\right|^{2},\left|U_{e 4}\right|^{2},\left|U_{e 5}\right|^{2}$ giving the results in Table 5. The angles $\theta_{14}$ and $\theta_{15}$ are taken from the best fit values of table 1. We take 


\begin{tabular}{|c|c|c|c|}
\hline Hierarchy & i & $P_{e i}^{m}$ & $P_{x i}^{m}$ \\
\hline \multirow[t]{5}{*}{$\overline{\mathrm{N} 2+\mathrm{N} 3}$} & 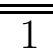 & 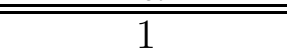 & 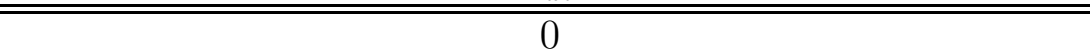 \\
\hline & 2 & 0 & $P_{25} P_{24}$ \\
\hline & 3 & 0 & $P_{25}\left(1-P_{24}\right)\left(1-P_{34}\right)+\left(1-P_{25}\right)\left(1-P_{35}\right) P_{34}+P_{35} P_{34}$ \\
\hline & 4 & 0 & $P_{25}\left(1-P_{24}\right) P_{34}+\left(1-P_{25}\right)\left(1-P_{35}\right)\left(1-P_{34}\right)+P_{35}\left(1-P_{34}\right)$ \\
\hline & 5 & 0 & $\left(1-P_{35}\right)+\left(1-P_{25}\right) P_{35}$ \\
\hline \multirow[t]{5}{*}{$\mathrm{N} 2+\mathrm{I} 3$} & 1 & $\overline{P_{13}}$ & $P_{35} P_{34}\left(1-P_{13}\right)$ \\
\hline & 2 & 0 & $P_{35}\left(1-P_{34}\right)\left(1-P_{24}\right)+\left(1-P_{35}\right)\left(1-P_{25}\right) P_{24}+P_{25} P_{24}$ \\
\hline & 3 & $1-P_{13}$ & $P_{35} P_{34} P_{13}$ \\
\hline & 4 & 0 & $P_{35}\left(1-P_{34}\right) P_{24}+\left(1-P_{35}\right)\left(1-P_{25}\right)\left(1-P_{24}\right)+P_{25}\left(1-P_{24}\right)$ \\
\hline & 5 & 0 & $\left(1-P_{25}\right)+\left(1-P_{35}\right) P_{25}$ \\
\hline \multirow[t]{5}{*}{$\mathrm{H} 2+\mathrm{N} 3$} & 1 & $P_{14}$ & 0 \\
\hline & 2 & 0 & $P_{25}$ \\
\hline & 3 & 0 & $P_{35}+\left(1-P_{25}\right)\left(1-P_{35}\right)$ \\
\hline & 4 & $1-P_{14}$ & 0 \\
\hline & 5 & 0 & $\left(1-P_{35}\right)+\left(1-P_{25}\right) P_{35}$ \\
\hline \multirow[t]{5}{*}{$\mathrm{H} 2+\mathrm{I} 3$} & 1 & $P_{14} P_{13}$ & $P_{35}\left(1-P_{13}\right)$ \\
\hline & 2 & 0 & $\left(1-P_{35}\right)\left(1-P_{25}\right)+P_{25}$ \\
\hline & 3 & $\left(1-P_{13}\right) P_{14}$ & $P_{35} P_{13}$ \\
\hline & 4 & $\left(1-P_{14}\right)$ & 0 \\
\hline & 5 & 0 & $\left(1-P_{25}\right)+\left(1-P_{35}\right) P_{25}$ \\
\hline \multirow[t]{5}{*}{$\mathrm{I} 2+\mathrm{N} 3$} & 1 & $P_{15} P_{14}$ & 0 \\
\hline & 2 & 0 & 1 \\
\hline & 3 & 0 & 1 \\
\hline & 4 & $P_{15}\left(1-P_{14}\right)$ & 0 \\
\hline & 5 & $1-P_{15}$ & 0 \\
\hline \multirow[t]{5}{*}{$\mathrm{I} 2+\mathrm{I} 3$} & 1 & $P_{15} P_{14} P_{13}$ & $1-P_{13}$ \\
\hline & 2 & 0 & 1 \\
\hline & 3 & $P_{15} P_{14}\left(1-P_{13}\right)$ & $P_{13}$ \\
\hline & 4 & $P_{15}\left(1-P_{14}\right)$ & 0 \\
\hline & 5 & $1-P_{15}$ & 0 \\
\hline
\end{tabular}

Table 4: The probabilities $P_{e i}^{m}$ and $P_{x i}^{m}$ for three active neutrinos plus two sterile neutrinos, where $P_{\alpha i}^{m}$ is given by Eq. (26) and $P_{i j}$ is the flip probability at the resonance between the $\nu_{i}$ and $\nu_{j}$ mass eigenstates. 


\begin{tabular}{|c|c|c|}
\hline Hierarchy & $F_{\text {noshock }}$ & $F_{\text {shock }}$ \\
\hline $\mathrm{N} 2+\mathrm{N} 3$ & $\left|U_{e 1}\right|^{2} F_{e}^{0}$ & $P_{24} P_{25}\left|U_{e 2}\right|^{2} F_{x}^{0}$ \\
$\mathrm{~N} 2+\mathrm{I} 3$ & $\left|U_{e 3}\right|^{2} F_{e}^{0}+\left(\left|U_{e 4}\right|^{2}+\left|U_{e 5}\right|^{2}\right) F_{x}^{0}$ & $\left|U_{e 1}\right|^{2} P_{13} F_{e}^{0}+\left(\left(P_{24}+P_{25}\right)\left|U_{e 2}\right|^{2}+P_{24} P_{25}\left(\left|U_{e 1}\right|^{2}-\left|U_{e 2}\right|^{2}\right)\right) F_{x}^{0}$ \\
$\mathrm{H} 2+\mathrm{N} 3$ & $\left|U_{e 4}\right|^{2} F_{e}^{0}+\left(\left|U_{e 3}\right|^{2}+\left|U_{e 5}\right|^{2}\right) F_{x}^{0}$ & $P_{24}\left|U_{e 1}\right|^{2} F_{e}^{0}+P_{25}\left|U_{e 2}\right|^{2} F_{x}^{0}$ \\
$\mathrm{H} 2+\mathrm{I} 3$ & $\left|U_{e 2}\right|^{2} F_{x}^{0}$ & $P_{25}\left|U_{e 1}\right|^{2} F_{x}^{0}$ \\
$\mathrm{I} 2+\mathrm{N} 3$ & $\left|U_{e 2}\right|^{2} F_{x}^{0}$ & $P_{24} P_{25}\left|U_{e 1}\right|^{2} F_{e}^{0}$ \\
$\mathrm{I} 2+\mathrm{I} 3$ & $\left(\left|U_{e 1}\right|^{2}+\left|U_{e 2}\right|^{2}\right) F_{x}^{0}$ & $-P_{13}\left|U_{e 1}\right|^{2} F_{x}^{0}$ \\
\hline
\end{tabular}

Table 5: The flux of neutrinos in the approximation that $\left|U_{e 1}\right|^{2},\left|U_{e 2}\right|^{2}>>\left|U_{e 3}\right|^{2},\left|U_{e 4}\right|^{2},\left|U_{e 5}\right|^{2}$ and $P_{1 i}, P_{3 i} \simeq P_{2 i}$, where $\mathrm{i}=4$ or 5 .

$\theta_{34}=\theta_{35}=10^{-3}$; note that these are not constrained from any data. Finally we take $\theta_{13}=0.142$, within the bounds from equation 2.3.3 and $\theta_{24}=0.12$ and $\theta_{25}=10^{-3}$, chosen such that the LSND mixing angle $\theta_{L S N D}$ is equal to it's best fit value, where $\sin ^{2}\left(2 \theta_{L S N D}\right)=4\left(U_{e 4} U_{\mu 4}+U_{e 5} U_{\mu 5}\right)^{2}$. These angles are large such that the transition probabilities change from being adiabatic to non-adiabatic as the shock wave passes through each resonance. The signal is dominated by detection of anti-neutrinos leaving the supernova in the first and second mass eigenstate. In all but one case the mixing angles are sufficiently small such that the multiple resonances that these anti-neutrinos cross are far enough apart such that they do not overlap and therefore each resonance can be considered as independent. The exception is the combination of resonances corresponding to $\Delta m_{43}^{2}$ and $\Delta m_{42}^{2}$, however numerical checks have shown that the total transition probability is still well represented by a product of the two flip probabilities. Using this we have computed the number of photons that would be detected in IceCube for a galactic supernova. Figs. 9, 10, 11] 12, 13] and 14 are for the LL case while Fig. 15 compares the impact of the change in the initial neutrino fluxes and spectra from LL to G1 and G2.

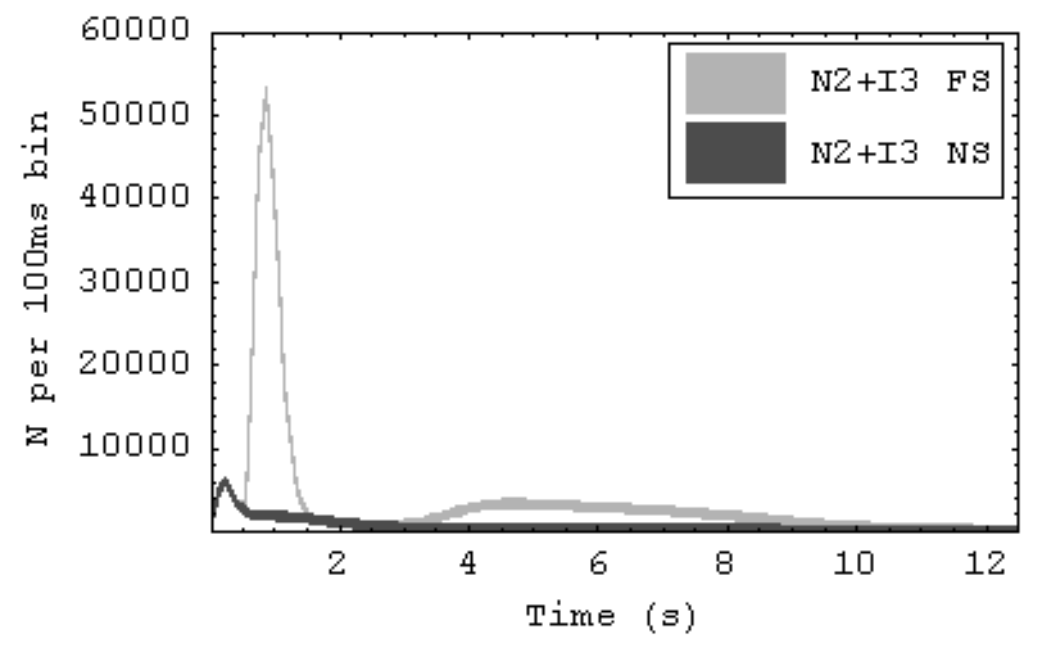

Figure 9: The number of photons detected for a N2+I3 mass hierarchy calculated for 100ms time bins with a forward shock (FS) and without a shock (NS). 
Fig. 9] shows the number of photons detected in time bins of $100 \mathrm{~ms}$, due to a supernova for neutrinos in a N2+I3 mass hierarchy, with and without a shock wave. There are bumps in the signal due to the propagation of the shock wave in the first $2 \mathrm{~s}$ and from $4 \mathrm{~s}-11 \mathrm{~s}$. As has been discussed for the case of just three active neutrinos, the latter structure is due to the propagation of the shockwave through the resonant density corresponding to the atmospheric mass splitting. The bumps in the first 1.5s is due to the propagation of the shockwave through the resonant densities corresponding to the sterile neutrinos.

We first concentrate on the $\Delta m_{31}^{2}$ driven "atmospheric resonance" to determine the changes due to the mixing with sterile neutrinos. The effect of the shock wave is only relevant if the three active neutrinos have an inverse hierarchy in their masses. This is evident from Table 5 , where the flip probability $P_{13}$ appears only for cases with I3. For these cases we show in Fig. [10 the number of photons detected on the same time bins as that shown in Fig. 7 . We do not show the case for $\mathrm{H} 2+\mathrm{I} 3$ because, c.f. Table [5] the shock wave only causes a change in the signal if it also passes through a sterile resonance at the same time. In theoretical simulations of the shockwave this does not happen and therefore no "bump" should be observed. From the Figs. [7 and 10] we see that the N2+I3 hierarchy gives an increase in the signal compared to the case of only 3 active neutrinos while the I2+I3 case shows a decrease. In both cases we get a statistically significant signal.

Turning to the sterile resonance region we present in Figs. 11, 12, 13] and 14] our numerical results for the sterile resonances. The structure of the signal spans of the order of $0.1 \mathrm{~s}$ and therefore a detector needs to have a time resolution of at least 10ms to try to disentangle the information. The number of bumps is determined by several factors. First, it is dependent on the mass hierarchy which determines the number of resonances which the neutrino passes through, (see Table 4). If a neutrino passes through two sterile resonances it could create two bumps; however if the resonances are close inside the supernova, only one broader bump may be observed. If the shockwave has a forward and reverse shock the double peak structure in the probability can cause several bumps in the signal, as can clearly be seen in Fig. 12 (c) and (d). For the N2+I3 and H2+N3 mass hierarchies, because $\left|U_{e 1}\right|^{2},\left|U_{e 2}\right|^{2}>>\left|U_{e 3}\right|^{2},\left|U_{e 4}\right|^{2},\left|U_{e 5}\right|^{2}$, the number of events in the absence of a shock is an order of magnitude smaller than that of other hierarchies. Therefore observation of a large ratio of the height of the bump to the background supernova signal would be characteristic of these mass hierarchies. However in practice this could be misidentified if the luminosity varied rapidly in time.

The effect of changing the average energy and total number flux of the neutrinos is shown in Fig. 15. The six panels are for the six different mass spectra and we consider the case where we have both forward and reverse shocks. We can see from the figure that changing the supernova neutrino model from LL to G1 or G2 does not wipe out any of the interesting structures in the signal.

\section{$5 \quad$ Summary and Conclusions}

Our analysis of the neutrino signals in the IceCube detector that would result from a "standard" supernova at $10 \mathrm{kpc}$ from earth has shown that oscillations to sterile neutrinos will be easily detectable for a wide variety of neutrino spectra. As may be seen from the figures, for $10 \mathrm{msec}$ time bins, the event rate is typically very large and supernovae as far as about $30 \mathrm{kpc}$ from 

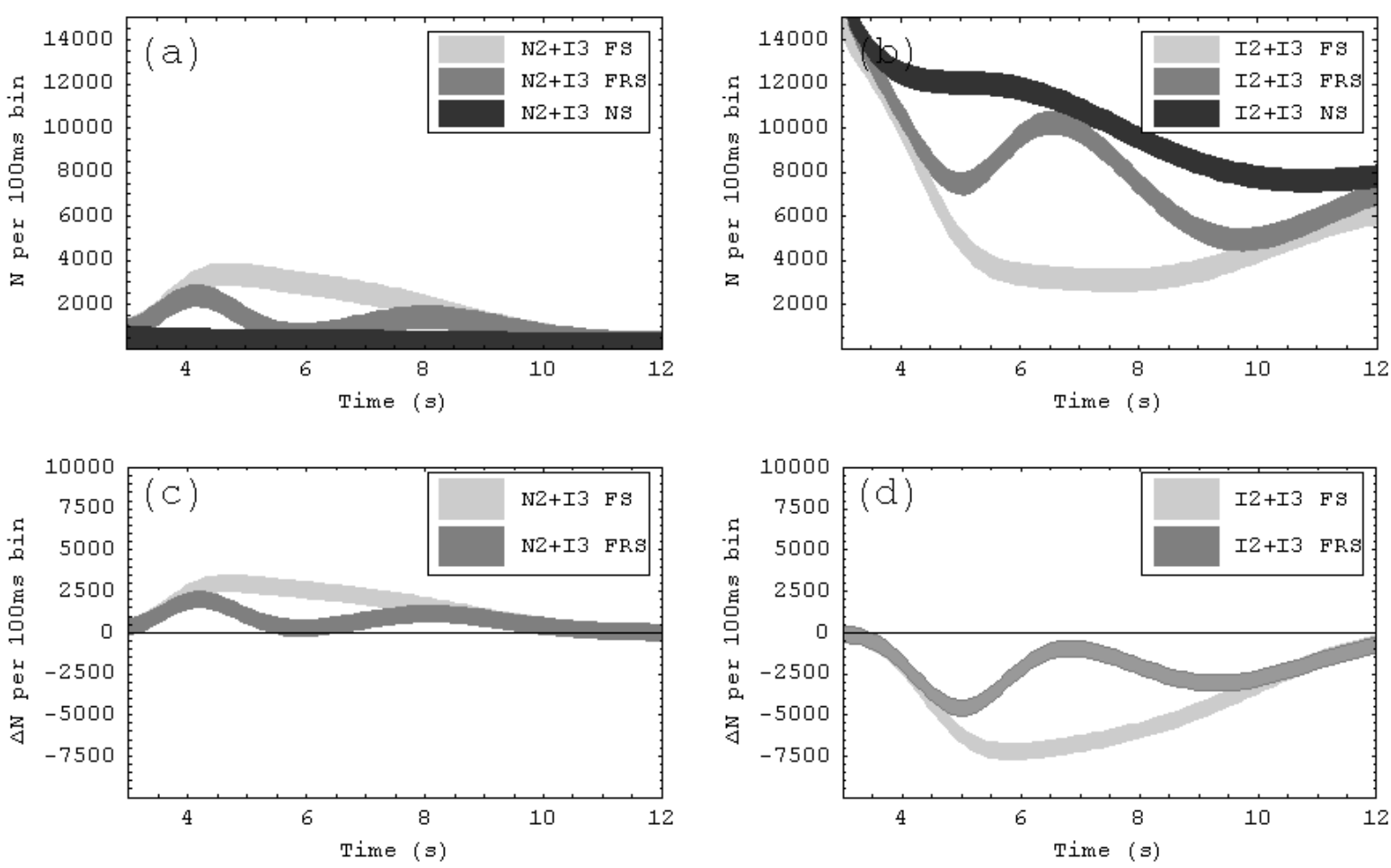

Figure 10: The number of additional photons detected in 100ms time bins, for the cases of a forward shock (FS), a forward and reverse shock (FRS) and no shock (NS) for the mass hierarchies:(a) N2+I3, (b) I2+I3. The lower panels show the corresponding difference in between the number of photons expected due to the effect of the shock for the mass hierarchies: (c) N2+I3, (d) $\mathrm{I} 2+\mathrm{I} 3$.

earth will still give observable effects. The reason the signals are so distinctive is a result of the combination of resonant conversion within the supernova with the time dependent structure resulting from the propagation of a shock wave through the supernova. Together this gives a characteristic time dependent signal that is readily observable with a detector time resolution better than $10 \mathrm{msec}$. Since oscillation to sterile neutrinos generates a disappearance event the results are relatively insensitive to uncertainties in the initial energy spectra and luminosities.

The signal coming from resonant effects involving just three antineutrinos with an inverted mass hierarchy is much more sensitive to the initial spectra and luminosities because the signal vanishes if both are degenerate for the three flavours. For the case the energy spectra differ significantly, as in the Lawrence Livermore simulation, the resulting signal is again clearly visible in IceCube. However if the energy spectra are degenerate, as recent studies seem to indicate, then one must rely on differences in the initial luminosities. To date no full simulation has been performed over the time period of interest but extrapolating the recent simulations, which extend up to $750 \mathrm{~ms}$, to later times suggests that even in this case the resonant conversion in the presence of a shock wave would be clearly visible despite a reduction of about a factor of two in the signal. To improve our confidence in this result it is clearly of importance to have a reliable 

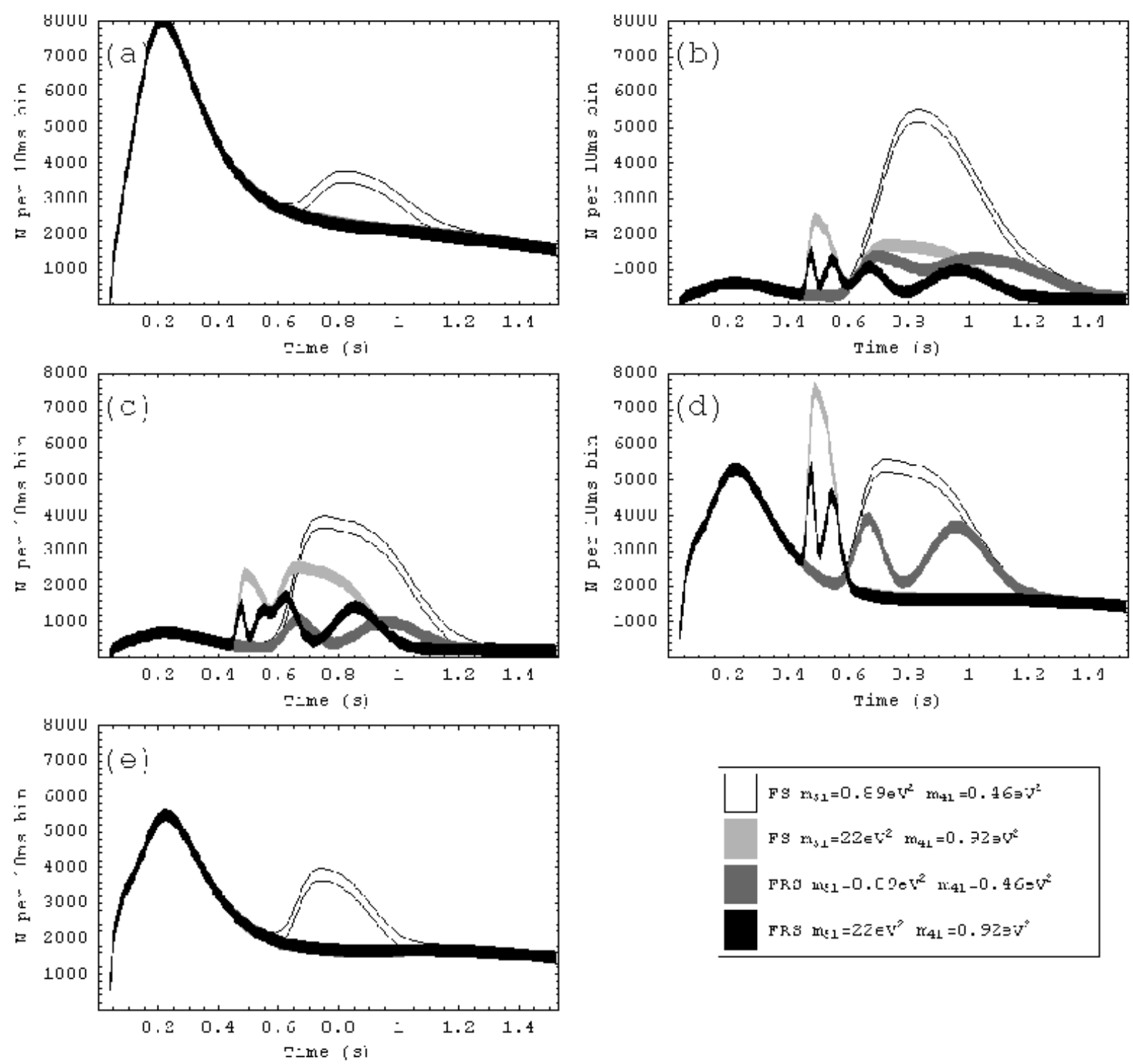

Figure 11: The number of photons expected in 10ms time bins, due to a forward shock (FS) and a forward and reverse shock (FRS), for the mass hierarchies:(a) N2+N3, (b) N2+I3, (c) H2+N3, (d) $\mathrm{H} 2+\mathrm{I} 3$, (e) I2+N3. We show the results for both the solutions given in Table 1 Note that the shock wave has no observable effect for $\mathrm{I} 2+\mathrm{I} 3$. 

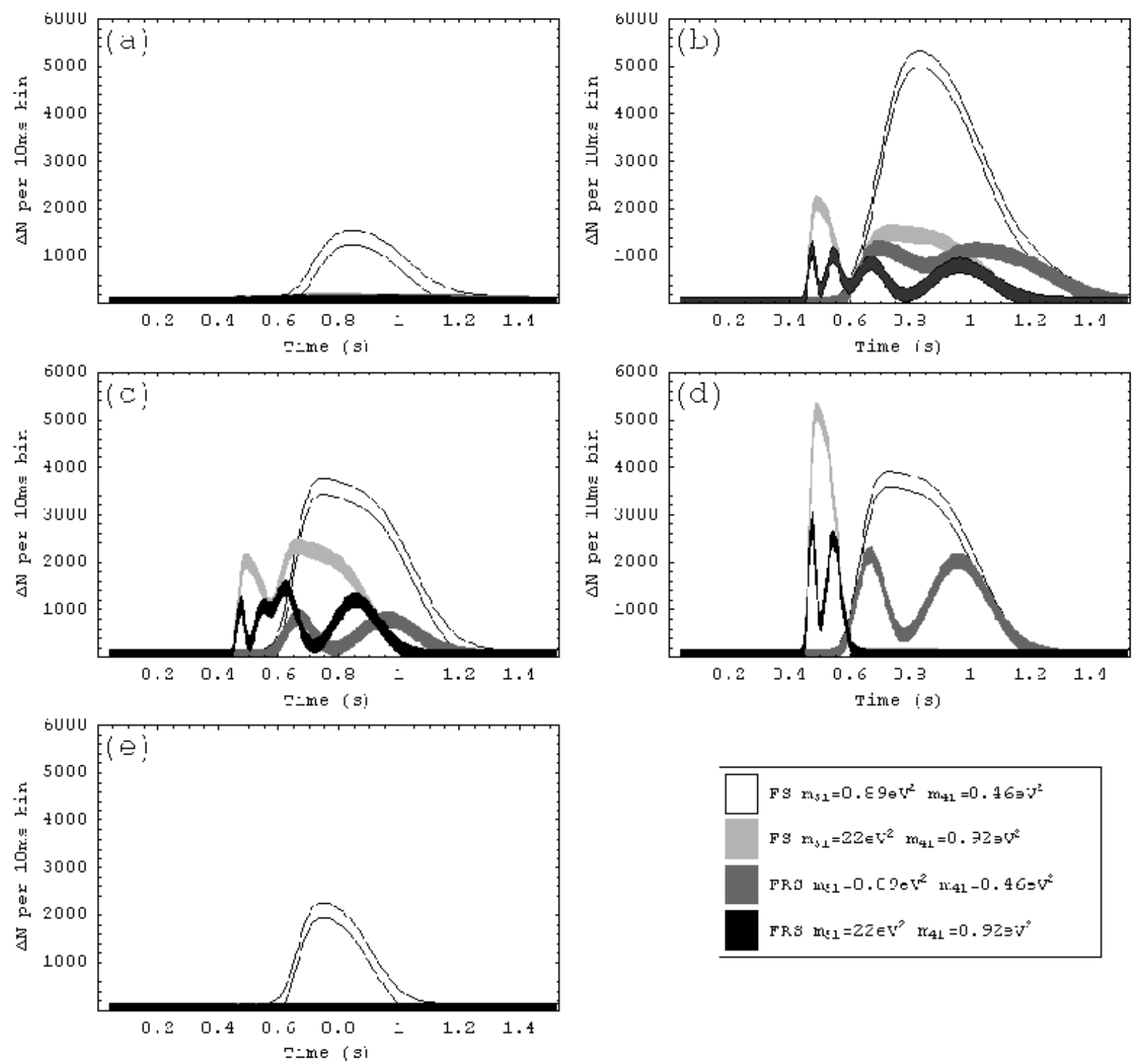

Figure 12: The difference in the number of expected photons in 10ms time bins due to the effect of a forward shock (FS) and a forward and reverse shock (FRS), for the mass hierarchies: (a) $\mathrm{N} 2+\mathrm{N} 3$, (b) N2+I3, (c) H2+N3, (d) H2+I3, (e) I2+N3. 

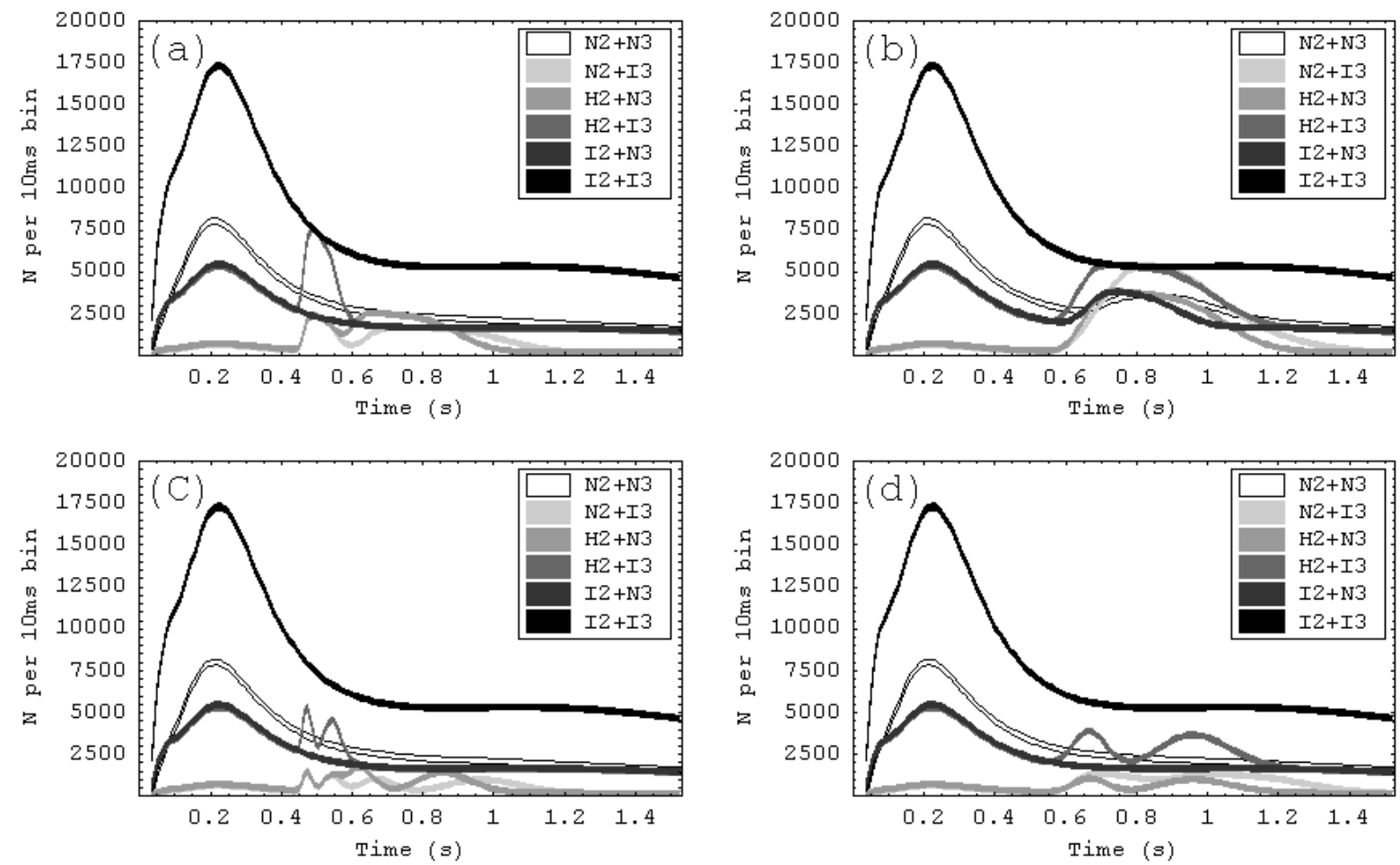

Figure 13: The number of photons detected in 10ms time bins due to the shockwave in various mass hierarchies, where (a) is for forward shock and $\Delta m_{51}^{2}=22 e V^{2}, \Delta m_{41}^{2}=0.92 e V^{2}$, (b) is for forward shock and $\Delta m_{51}^{2}=0.89 \mathrm{eV}^{2}, \Delta m_{41}^{2}=0.46 \mathrm{eV}^{2}$, (c) is for forward and reverse shock and $\Delta m_{51}^{2}=22 \mathrm{eV}^{2}, \Delta m_{41}^{2}=0.92 \mathrm{eV}^{2},(\mathrm{~d})$ is for forward and reverse shock and $\Delta m_{51}^{2}=0.89 \mathrm{eV}^{2}$, $\Delta m_{41}^{2}=0.46 \mathrm{eV}^{2}$. 

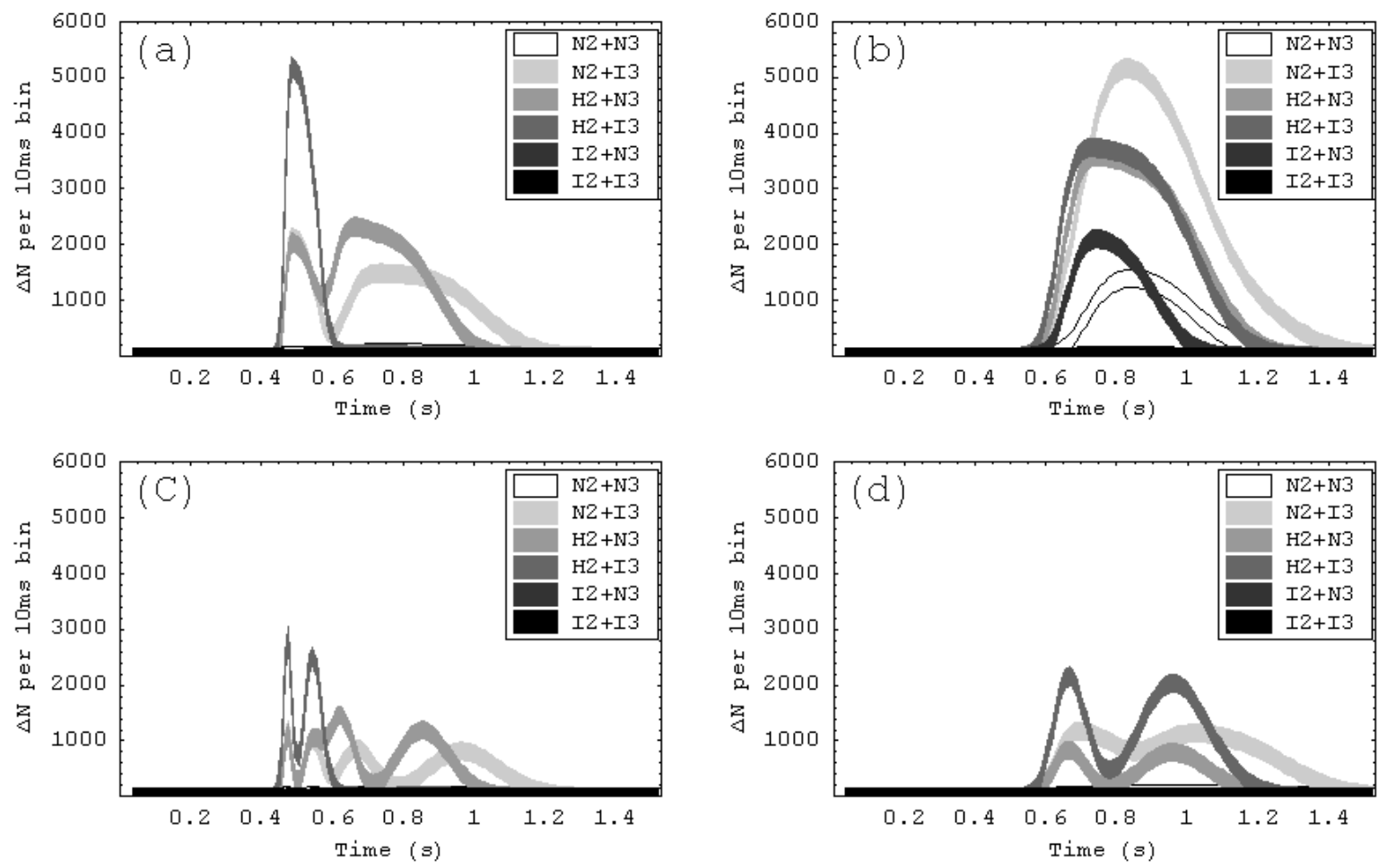

Figure 14: The number of additional photons detected in 10ms time bins due to the shockwave in various mass hierarchies, where (a) is for forward shock and $\Delta m_{51}^{2}=22 \mathrm{eV}^{2}, \Delta m_{41}^{2}=0.92 \mathrm{eV}^{2}$, (b) is for forward shock and $\Delta m_{51}^{2}=0.89 \mathrm{eV}^{2}, \Delta m_{41}^{2}=0.46 \mathrm{eV}^{2}$, (c) is for forward and reverse shock and $\Delta m_{51}^{2}=22 \mathrm{eV}^{2}, \Delta m_{41}^{2}=0.92 \mathrm{eV}^{2}$, (d) is for forward and reverse shock and $\Delta m_{51}^{2}=$ $0.89 \mathrm{eV}^{2}, \Delta m_{41}^{2}=0.46 \mathrm{eV}^{2}$. 

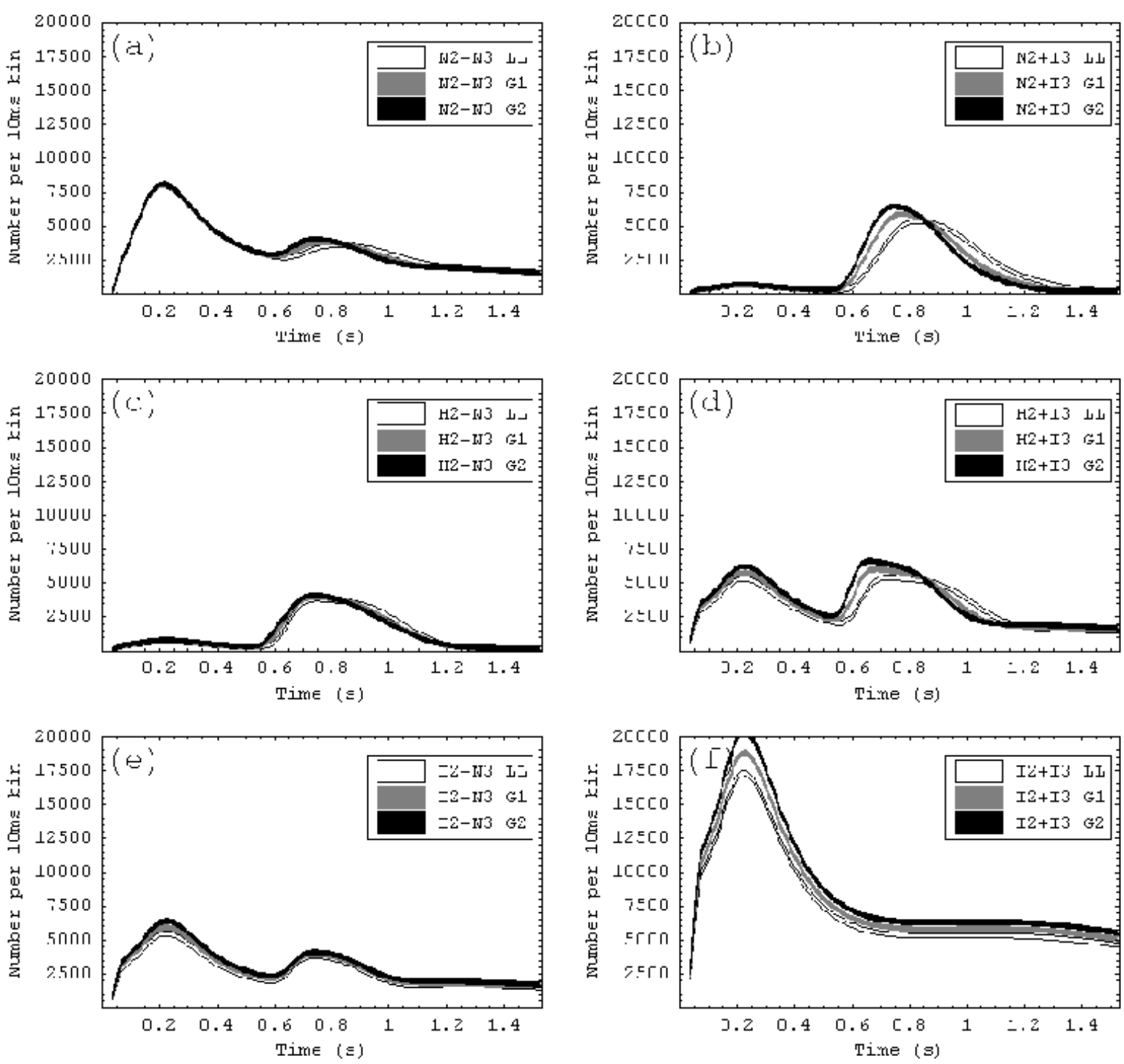

Figure 15: Effect of changing the supernova neutrino model on the resultant signal in the detector. The number of photons expected in 10ms time bins, due to a forward shock and $\Delta m_{51}^{2}=0.89 \mathrm{eV}^{2}, \Delta m_{41}^{2}=0.46 \mathrm{eV}^{2}$, for the mass hierarchies: (a) N2+N3, (b) N2+I3, (c) $\mathrm{H} 2+\mathrm{N} 3$, (d) $\mathrm{H} 2+\mathrm{I} 3$, (e) $\mathrm{I} 2+\mathrm{N} 3$ (f) $\mathrm{I} 2+\mathrm{I} 3$. 
simulation of neutrino propagation through the supernova over a 10 second period including all the significant antineutrino processes.

Observation of sharp time dependent neutrino oscillation signals will also provide a probe of the propagation of the shock wave through the supernova. As our simulations show it is possible that one can see both the forward and reverse shocks and the structure of the signal will give information about their spatial structure. At present our knowledge about the nature of the shock is quite primitive and clearly better simulations of the shock properties will improve our ability to interpret the signals. A very recent paper 16 has pointed out that turbulence after the shock can significantly affect the signals, broadening the structure, and clearly it is also of importance to study this in more detail.

In summary we have found that the detailed time structure of the neutrinos coming from supernovae can provide information, not accessible by other means, both on the mass spectrum and mixing angles of neutrinos and on supernovae seismology. This applies to those neutrino mass hierarchies and mixing angles, both for three active neutrinos and for a combination of active and sterile neutrinos, which give rise to resonant transitions within the supernovae. For the case involving sterile neutrinos our analysis has concentrated on the parameter space which can explain all neutrino oscillation phenomena including the LSND measurement. However, even if the LSND result should not be confirmed, the sensitivity of experiments such as IceCube is such that they will still be able to probe for a sterile neutrino component not otherwise observable.

\section{Acknowledgment}

We wish to thank Subir Sarkar for discussions. This work was partially supported by the EC 6th Framework Programme MRTN-CT-2004-503369, and a PPARC studentship.

\section{Appendix: Detector properties}

The following analysis is the same as that presented by Dighe, Keil and Raffelt [12. For simplicity only events detected by the $\bar{\nu}_{e} p \rightarrow n e^{+}$are considered. Each $e^{+}$emits Cerenkov light according to

$$
\frac{d^{2} N_{\gamma}}{d x d \lambda}=\frac{2 \pi \alpha \sin ^{2} \theta}{\lambda^{2}}
$$

where $N_{\gamma}$ is the number of emitted photons $x$ is the distance traveled by the $e^{+}, \lambda$ is the wavelength of the emitted photons, $\alpha$ is the fine structure constant and $\theta$ is the angle of the emitted photons to the direction of the $e^{+}$such that $\cos \theta=(n \beta)^{-1}$, where $\mathrm{n}$ is the refractive index of the ice $\left(n_{i c e}=1.31\right)$ and $\beta$ is the ratio of the speed of the positron in the medium to that in a vacuum $\left(\beta_{i c e} \sim 1\right)$. Integrating over the observable wavelengths, taking the mean free path of a positron to be $12 \mathrm{~cm}$ for an energy of $20 \mathrm{MeV}$ and assuming the two are proportional the number of photons emitted between observable wavelengths is:

$$
\frac{N_{\gamma}}{E_{\bar{\nu}_{e}}}=191 \mathrm{MeV}^{-1} f_{C h}
$$


where $E_{\bar{\nu}_{e}}$ is the energy of the $\bar{\nu}_{e}$ and $f_{C h}$ is a fudge factor. The number of detected photons per optical module is:

$$
\begin{gathered}
N_{\text {det }}=\frac{47.75}{M e V m^{-3}} f_{C h} f_{a b s} f_{O M} \rho \frac{1}{4 \pi D^{2}} \int_{t_{\min }}^{t_{\max }} d t \int_{0}^{\infty} d E \sigma(E) F(E, t) E \\
f_{O M}=\frac{Q}{0.20} \frac{A_{c a t}}{250 \mathrm{~cm}^{2}} \frac{\Omega_{a c c}}{2 \pi} \\
f_{a b s}=R_{a b s} / 100 \mathrm{~m}
\end{gathered}
$$

where $\rho=6.18 \times 10^{25} \mathrm{~m}^{-3}$ is the density of targets in ice, $\sigma$ (in $\mathrm{cm}^{2}$ ) is the cross section for $\bar{\nu}_{e} p \rightarrow n e^{+}, E$ (in MeV) is energy, $\mathrm{D}\left(\mathrm{cm}^{2}\right)$ is the distance to the supernova, $\mathrm{t}$ (in s) is time, $\mathrm{F}$ (in $\mathrm{MeV}^{-1} \mathrm{~s}^{-1}$ ) is the flux of anti-electron neutrinos, $Q$ is the average quantum efficiency of the detector, $A_{\text {cat }}$ is the effective photo cathode detection area, $\Omega_{a c c}$ is the angular acceptance range and $R_{a b s}$ is the absorption length of photons by the ice. 


\section{References}

[1] C. Athanassopoulos et al., (The LSND Collaboration) Phys. Rev. Lett. 77, 3082 (1996); C. Athanassopoulos et al., (The LSND Collaboration) Phys. Rev. Lett. 81, 1774 (1998).

[2] J. J. Gomez-Cadenas and M. C. Gonzalez-Garcia, Z. Phys. C 71, 443 (1996); S. Goswami, Phys. Rev. D 55, 2931 (1997). S. M. Bilenky, C. Giunti and W. Grimus, Eur. Phys. J. C 1, 247 (1998).

[3] B. T. Cleveland et al., Astrophys. J. 496, 505 (1998); J. N. Abdurashitov et al. [SAGE Collaboration], J. Exp. Theor. Phys. 95, 181 (2002); [Zh. Eksp. Teor. Fiz. 122, 211 (2002)] W. Hampel et al. [GALLEX Collaboration], Phys. Lett. B 447, 127 (1999); C. Cattadori, Talk at Neutrino 2004, Paris, France, June 14-19, 2004; S. Fukuda et al. [Super-Kamiokande Collaboration], Phys. Lett. B 539, 179 (2002); B. Aharmim et al. [SNO Collaboration], Phys. Rev. C 72, 055502 (2005).

[4] T. Araki et al. [KamLAND Collaboration], Phys. Rev. Lett. 94, 081801 (2005).

[5] Y. Ashie et al. [Super-Kamiokande Collaboration], Phys. Rev. D 71, 112005 (2005).

[6] E. Aliu et al. [K2K Collaboration], Phys. Rev. Lett. 94, 081802 (2005).

[7] M. Apollonio et al., Eur. Phys. J. C 27, 331 (2003); F. Boehm et al., Phys. Rev. D 64, 112001 (2001).

[8] Y. Declais et al., Phys. Lett. B 338, 383 (1994); F. Dydak et al., Phys. Lett. B 134, 281 (1984); I. E. Stockdale et al., Phys. Rev. Lett. 52, 1384 (1984); B. Armbruster et al. [KARMEN Collaboration], Phys. Rev. D 65, 112001 (2002); P. Astier et al. [NOMAD Collaboration], Phys. Lett. B 570, 19 (2003).

[9] M. Maltoni, et al., New J. Phys. 6, 122 (2004), hep-ph/0405172 v7.

[10] M. Sorel, J. M. Conrad and M. H. Shaevitz, Phys. Rev. D 70, 073004 (2004).

[11] L. Wolfenstein, Phys. Rev. D 17, 2369 (1978); S. P. Mikheev and A. Y. Smirnov, Sov. J. Nucl. Phys. 42 (1985) 913 [Yad. Fiz. 42, 1441 (1985)]; S. P. Mikheev and A. Y. Smirnov, Sov. J. Nucl. Phys. 42 (1985) 913 [Yad. Fiz. 42, 1441 (1985)].

[12] A. S. Dighe and A. Y. Smirnov, Phys. Rev. D 62, 033007 (2000); C. Lunardini and A. Y. Smirnov, JCAP 0306, 009 (2003); C. Lunardini and A. Y. Smirnov, Nucl. Phys. B 616, 307 (2001); K. Takahashi, M. Watanabe, K. Sato and T. Totani, Phys. Rev. D 64, 093004 (2001); K. Takahashi and K. Sato, Phys. Rev. D 66, 033006 (2002); S. Choubey, D. Majumdar and K. Kar, J. Phys. G 25, 1001 (1999); G. Dutta, D. Indumathi, M. V. N. Murthy and G. Rajasekaran, Phys. Rev. D 61, 013009 (2000); I. GilBotella and A. Rubbia, JCAP 0310, 009 (2003); A. S. Dighe, M. T. Keil and G. G. Raffelt, JCAP 0306, 006 (2003); A. S. Dighe et al., JCAP 0401, 004 (2004); H. Minakata et al., Phys. Lett. B 542, 239 (2002) A. Bandyopadhyay, S. Choubey, S. Goswami and K. Kar, arXiv:hep-ph/0312315. S. H. Chiu and T. K. Kuo, arXiv:hep-ph/0511345. 
[13] G. Dutta, D. Indumathi, M. V. N. Murthy, and G. Rajasekaran, Phys. Rev. D 62, 093014 (2000); G. Dutta, D. Indumathi, M. V. N. Murthy, and G. Rajasekaran, Phys. Rev. D 64, 073011 (2001); M. Sorel and J. Conrad, Phys. Rev. D 66, 033009 (2002); J. Fetter et al., Astropart. Phys. 18, 433 (2003).

[14] R. C. Schirato, G. M. Fuller, arXiv:astro-ph/0205390

[15] R. Tomas, M. Kachelriess, G. Raffelt, A. Dighe, H. T. Janka and L. Scheck, JCAP 0409, 015 (2004).

[16] G. L. Fogli, E. Lisi, A. Mirizzi and D. Montanino, arXiv:hep-ph/0603033.

[17] A. Achterberg et al. [IceCube Collaboration], arXiv:astro-ph/0604450, S. R. Klein[for the IceCube Collaboration], arXiv:astro-ph/0601269. K. Rawlins et al. [IceCube Collaboration], "IceCube: A Multipurpose Neutrino Telescope" http://icecube.wisc.edu

[18] G. L. Fogli, E. Lisi, A. Mirizzi and D. Montanino, Phys. Rev. D 68, 033005 (2003).

[19] V. Barger, P. Huber and D. Marfatia, Phys. Lett. B 617, 167 (2005).

[20] G. L. Fogli, E. Lisi, A. Mirizzi and D. Montanino, JCAP 0504, 002 (2005).

[21] A. S. Dighe, M. T. Keil and G. G. Raffelt, JCAP 0306, 005 (2003).

[22] K. Takahashi, M. Watanabe, K. Sato and T. Totani, Phys. Rev. D 64, 093004 (2001).

[23] S. Hannestad and G. Raffelt, Astrophys. J. 507, 339 (1998); R. Buras et al., Astrophys. J. 587, 320 (2003); M. T. Keil, G. G. Raffelt and H. T. Janka, Astrophys. J. 590, 971 (2003); M. Liebendoerfer et al., Astrophys. J. 620, 840 (2005). G. G. Raffelt, M. T. Keil, R. Buras, H. T. Janka and M. Rampp, arXiv:astro-ph/0303226. G. G. Raffelt, M. T. Keil, R. Buras, H. T. Janka and M. Rampp arXiv:astro-ph0303226.

[24] E. Lisi, A. Marrone, D. Montanino, A. Palazzo and S. T. Petcov, Phys. Rev. D 63, 093002 (2001); G. L. Fogli, E. Lisi, D. Montanino and A. Palazzo, Phys. Rev. D 65, 073008 (2002); A. Friedland, Phys. Rev. D 64, 013008 (2001).

[25] B. Dasgupta and A. Dighe, arXiv:hep-ph/0510219.

[26] T. K. Kuo and J. Pantaleone, Rev. Mod. Phys. 61, 937 (1989); T. K. Kuo and J. Pantaleone, Phys. Rev. D 37, 298 (1988).

[27] A. Bandyopadhyay et al., Phys. Lett. B 608, 115 (2005); S. Goswami, A. Bandyopadhyay and S. Choubey, Nucl. Phys. Proc. Suppl. 143, 121 (2005).

[28] S. Choubey, arXiv:hep-ph/0509217.

[29] G. L. Fogli, E. Lisi, A. Marrone and A. Palazzo, arXiv:hep-ph/0506083.

[30] S. Hannestad, Prog. Part. Nucl. Phys. 57, 309 (2006) arXiv:astro-ph/0511595; S. Hannestad, JCAP 0601, 001 (2006) arXiv:astro-ph/0510582. 
[31] M. T. Keil, arXiv:astro-ph/0308228, M. T. Keil, G. G. Raffelt and H. T. Janka, Astrophys. J. 590, 971 (2003). 